\title{
MD-GAN Based UAV Trajectory and Power Optimization for Cognitive Covert Communications
}

\author{
Zan Li, Senior Member, IEEE, Xiaomin Liao, Jia Shi, Member, IEEE, Xuan Xue, Li Li, Pei Xiao, Senior \\ Member, IEEE
}

\begin{abstract}
This paper investigates the covert performance for an unmanned aerial vehicle (UAV) jammer assisted cognitive radio network. In particular, the covert transmission of secondary users can be effectively protected by UAV jamming against the eavesdropping. For practical consideration, the UAV is assumed to only know certain partial channel distribution information (CDI), whereas not to know the detection threshold of eavesdropper. For this sake, we propose a model-driven generative adversarial network (MD-GAN) assisted optimization framework, consisting of a generator and a discriminator, where the unknown channel information and the detection threshold are learned weights. Then a GAN based joint trajectory and power optimization (GAN-JTP) algorithm is developed to train the MD-GAN optimization framework for covert communication, which results in the joint solution of UAV's trajectory and transmit power to maximize the covert rate and the probability of detection errors. Our simulation results show that, the proposed GAN-JTP with a rapid convergence speed can attain near-optimal solutions of UAV's trajectory and transmit power for the covert communication.
\end{abstract}

Index Terms-Covert communication, UAV jammer, generative adversarial network.

\section{INTRODUCTION}

$\mathrm{C}$ GNITIVE radio (CR) has been recognized as a promising technique to mitigate spectrum scarcity problem for future wireless networks. For instance, in Internet of Things (IoT) scenes, such as smart manufacturing, precision agriculture, healthcare, and military communication $[1,2]$. By applying CR capabilities, the CR based IoT (CR-IoT) network is established, where spectrum efficiency is maximized by intelligent spectrum sensing and dynamic access $[3,4]$. However, due to the dynamical accessing mechanism and the openness of wireless medium, legitimate CR users are vulnerable to potential malicious attacks, which incur severe CR security issue [5].

Copyright (c) 2021 IEEE. Personal use of this material is permitted. However, permission to use this material for any other purposes must be obtained from the IEEE by sending a request to pubs-permissions@ieee.org.

This work was supported in part by the Key Project of National Natural Science Foundation of China under Grant 61631015, in part by the Nationa Natural Science Foundation for Distinguished Young Scholar of China under Grant 61825104, in part by National Natural Science Foundation of China under Grant 61941105 and 61901327

Z. Li, X. Liao, J. Shi, and X. Xue are with the State Key Laboratory of Integrated Service Networks, School of Telecommunications Engineering, Xidian University, Xi' an 710071, China. X. Liao is also with the School of Information and Communications, National University of Defense Technology, Xi'an 710106, China. (E-mail: zanli@xidian.edu.cn, 1xm8410@163.com, jiashi@xidian.edu.cn, xuanx@xidian.edu.cn).

L. Li is with the Provincial Key Laboratory of Information Coding and Transmission, Southwest Jiaotong University, Chengdu 610031, China. (Email: 115e08@swjtu.edu.cn).

P. Xiao is with the Institute for Communication Systems (ICS), Home for 5G \& 6G Innovation Centres, University of Surrey, Guildford, Surrey, GU2 7XH, United Kingdom. (E-mail: p.xiao@surrey.ac.uk).
Traditional security techniques focus on information protection against eavesdropping, such as cryptographic approaches [6]. However, they demand high computational complexity for key distribution and management, and could be deciphered by malicious computing resources. On the other hand, physicallayer security (PLS) exploits the dynamic characteristics of wireless medium to minimize the information obtained by eavesdroppers [7,8]. Unfortunately, PLS mechanism highly relies on hiding the physical layer patterns of wireless nodes, which may be exposed to adversaries. On the basis of information protection, future cognitive radio networks (CRNs) will demand a higher level of security, i.e. protecting communication behavior, which is referred to as an emerging low detection/intercept-probability communication paradigm, a.k.a. covert communication [9]. For instance, in a military operation, once the adversary discerns that a wireless transmission is taking place, network attacks will be carried out, thus hiding the communication process is highly desirable. Apparently, compared with the physical-layer security preventing an adversary from determining the content of the message, covert communication aims to hide the presence of a wireless transmission and achieve higher level of security, thus has attracted significant research interests.

\section{A. Related Works}

The fundamental bounds on covert communications have been studied over additive white Gaussian noise (AWGN) channels [10,11], binary symmetric channels (BSC) [12], and discrete memoryless channels (DMC) [13], respectively. Specifically, a square root law has been proved in [10]: $\mathcal{O}(\sqrt{n})$ bits of information can be transmitted to a legitimate receiver reliably when channel uses $n \rightarrow \infty$. In addition, a positive covert rate can be guaranteed by applying certain approaches, such as relay schemes [14], transmit power control [15], and cooperative jamming [16-19]. In particular, cooperative jamming is the most efficient way, where external artificial noise (AN) is emitted to produce uncertainty at eavesdropping side. Note that, the transmit power of AN should be managed carefully in order to enhance covert communications [20]. Nevertheless, the existing cooperative jamming schemes mainly considered static jammers which become less effective if malicious terminals are located far away.

Therefore, due to the flexible deployment and controllable mobility, unmanned aerial vehicle (UAV) has been proposed as a new paradigm to facilitate fast and highly flexible deployment of communication infrastructure with sustainable services [21, 
22]. On the other hand, UAV can also offer a new opportunity to perform mobile jamming so as to further improve the secure performance, e.g. secure transmission assisted by PLS technique [23-25]. Nevertheless, UAV-based secure communications are still facing some key challenges, such as how to balance secrecy rate maximization [26], and energy efficiency maximization [27]. On the other hand, the only one work [28] investigated the approximate expression of privacy rate under both the AWGN and Nakagami-m fading scenarios by employing UAV-jammer for covert communication.

Nevertheless, in order to acquire channel information, the aforementioned works, including PLS technique and covert communication, have to leverage signaling interchange between UAV and transmitter. Moreover, the UAV should know the warden's detection threshold in advance [28, 29]. But these requirements are very impractical in covert communication scenarios. Therefore, this motivates us to study the UAV jammer aided covert communication upon having partial channel distribution information (CDI) knowledge and not having detection threshold used by eavesdropper.

\section{B. Motivations and Contributions}

It is extremely difficult for traditional approaches, such as convex optimization, to solve the joint optimization problem of UAV's trajectory and transmit power when missing the detection threshold and full channel information. By contrast, modeldriven generative adversarial network (MD-GAN) approach has become a new research trend to solve the optimization problem with limited priori information in the application of artificial intelligence [30,31], and it is able to provide the feasibility and effectiveness for our UAV jammer aided cognitive radio system under non-ideal scenarios. Motivated by the above, in this paper we commence a pioneer work on blending the advantages of the MD-GAN and UAV jamming for covert communications. The main contributions of our paper are summarized as follows.

- We develop a covert communication system model, where the UAV playing as the cooperative jammer helps cognitive covert transmission for maximizing the covert rate at the legitimate receiver and the probability of detection errors at the eavesdropper. In particular, we study a practical setup: the joint optimization for UAV's trajectory and transmit power under the assumptions of knowing the partial CDI and not knowing the detection threshold of the malicious terminal. Furthermore, we formulate and carry out a comprehensive analysis for the multi-objective nonconvex optimization problem, which transforms the whole optimization process into a dynamic game between the UAV and the imaginary malicious terminal.

- We build a MD-GAN optimization framework, where the number of layers, the number of neurons, the weights and non-linear transforms of neurons per layer are designed by the model-driven method. Furthermore, in our MDGAN framework, the unknown channel coefficients and the detection threshold are treated as the trained weights. Moreover, we prove that, the proposed MD-GAN framework can provide a promising solution to the transformed dynamic game, and can present communication infrastructures determined, in the premise of limited training data.
- To train the MD-GAN optimization framework with limited labeling data, we propose a joint trajectory and power optimization algorithm, namely GAN-JTP, for the UAVenabled cooperative jamming. Specifically, the proposed GAN-JTP algorithm alternatively trains the generator and the discriminator in a competitive manner, and is capable of obtaining the Nash equilibrium within a relatively small number of iterations.

- We carry out a comprehensive performance analysis for our UAV-jammer assisted covert cognitive radio system when employing the proposed GAN-JTP algorithm. Our simulation results show that, the proposed GAN-JTP with a rapid convergence speed can attain near-optimal solutions of UAV's trajectory and transmit power for the covert communication. Therefore, the GAN-JTP algorithm enabling UAV jamming constitutes a promising candidate that facilitates covert communications for secondary users in cooperative CRNs.

\section{System Model AND Problem Formulation}

\section{A. System Model}

Fig. 1 depicts a CRN consisting of a transmit-receive pair of primary users and that of secondary users, which are denoted by PT, PR, ST, and SR, respectively. In particular, underlay strategy is used for spectrum accessing. Further, there exists an eavesdropper, Eve, randomly distributed in a certain area, who is a malicious competitor or adversary and wants to detect the underlay transmission of the secondary users. However, for the sake of covert communication, an UAV sends jamming signal to confuse the detection of eavesdropper.

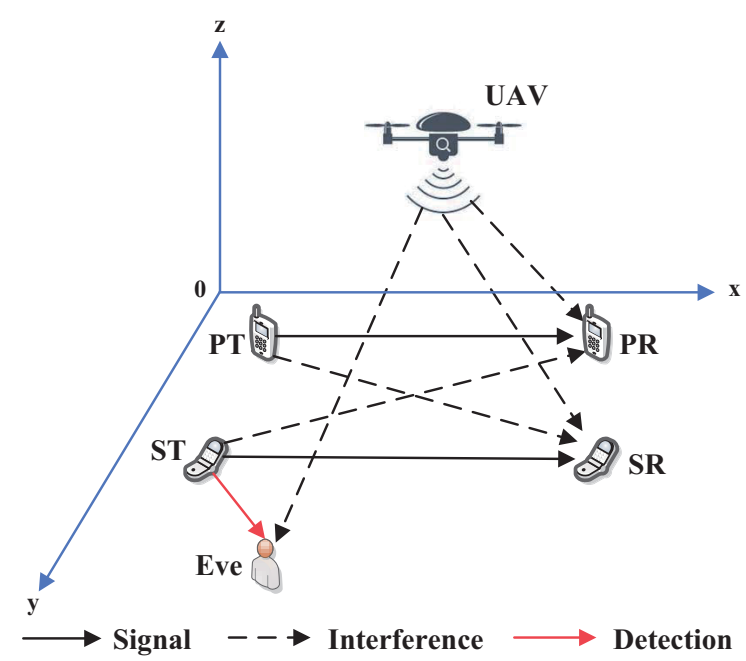

Fig. 1. Schematic for covert communication in the context of UAV-enabled cooperative jamming system.

Specially, PT, PR, ST, and SR on the ground have the locations of three-dimensional (3D) coordinates $\left(x^{\mathrm{PT}}, y^{\mathrm{PT}}, 0\right)$, $\left(x^{\mathrm{PR}}, y^{\mathrm{PR}}, 0\right),\left(x^{\mathrm{ST}}, y^{\mathrm{ST}}, 0\right)$, and $\left(x^{\mathrm{SR}}, y^{\mathrm{SR}}, 0\right)$, which are assumed to be known by the UAV. In practice, UAV knows Eve's approximate location by detecting the signals emitted from Eve, or by monitoring the potential information leakage [32]. For 
practical consideration, the Gaussian error model is employed to model Eve's location errors [29], leading to

$$
x^{\text {Eve }}=\hat{x}^{\text {Eve }}+\Delta x^{\text {Eve }}, y^{\text {Eve }}=\hat{y}^{\text {Eve }}+\Delta y^{\text {Eve }}, z^{\text {Eve }}=0 .
$$

Note that, Eve's coordinates $\hat{x}^{\text {Eve }}$ and $\hat{y}^{\text {Eve }}$ are estimated from UAV side, where $\Delta x^{\text {Eve }}$ and $\Delta y^{\text {Eve }}$ are estimation errors following the distribution of $\mathcal{N}\left(0, \varepsilon_{\text {Eve }}^{2}\right)$.

Furthermore, it is assumed that the UAV operates for a finite time period $T$, which can be equally divided into $N$ time slots, each of which is denoted by $\delta=\frac{T}{N}$. Let us assume that, the location of UAV is approximately static within each time slot, and the coordinate of UAV becomes $\left(x^{\mathrm{UAV}}[n], y^{\mathrm{UAV}}[n], z^{\mathrm{UAV}}[n]\right), n \in\{1, \ldots, N\}$. Further, the mobility constraints of UAV can be formulated as

$$
\begin{aligned}
& \left(x^{\mathrm{UAV}}[n]-x^{\mathrm{UAV}}[n-1]\right)^{2}+\left(y^{\mathrm{UAV}}[n]-y^{\mathrm{UAV}}[n-1]\right)^{2}+\left(z^{\mathrm{UAV}}[n]\right. \\
& \left.-z^{\mathrm{UAV}}[n-1]\right)^{2} \leq\left(V_{\max } \delta\right)^{2}, n \in\{2,3, \ldots, N\}, \\
& z^{\mathrm{UAV}}[n] \geq H_{\min }, n \in\{1,2,3, \ldots, N\},
\end{aligned}
$$

where $V_{\max }$ is the maximum flight speed in each time slot, $H_{\text {min }}$ denotes the minimum flight altitude. Let us denote the takeoff and landing locations by $\left(x_{1}^{\mathrm{UAV}}, y_{1}^{\mathrm{UAV}}, z_{1}^{\mathrm{UAV}}\right)$ and $\left(x_{N}^{\mathrm{UAV}}, y_{N}^{\mathrm{UAV}}, z_{N}^{\mathrm{UAV}}\right)$, respectively.

We assume each node is equipped with a single antenna ${ }^{1}$. The channel gains for the links of PT-PR, PT-SR, ST-PR, STSR, ST-Eve, UAV-PR, UAV-SR, and UAV-Eve are denoted as $h^{\mathrm{PT}, \mathrm{PR}}, h^{\mathrm{PT}, \mathrm{SR}}, h^{\mathrm{ST}, \mathrm{PR}}, h^{\mathrm{ST}, \mathrm{SR}}, h^{\mathrm{ST}, \mathrm{Eve}}, h^{\mathrm{UAV}, \mathrm{PR}}, h^{\mathrm{UAV}, \mathrm{SR}}$, and $h^{\mathrm{UAV} \text {,Eve }}$, respectively. In addition, in order to evaluate the lower-bound performance of our covert communication, we consider the worst case, where both terrestrial channels and air-to-ground channels are mainly dominated by the non-lineof-sight (NLoS) paths with Rayleigh scattering [33,34]. More specifically, the channel gain between each transceiver in $n$th $(n \in\{1, \ldots, N\})$ time slot can be given by

$$
h^{a, b}[n]=\sqrt{\frac{\rho}{d_{a, b}^{\alpha_{0}}[n]}} g^{a, b}[n],, a, b \in\{\mathrm{PT}, \mathrm{PR}, \mathrm{ST}, \mathrm{SR}, \mathrm{Eve}, \mathrm{UAV}\},
$$

where $d_{a, b}[n]=\sqrt{\left(x^{a}[n]-x^{b}[n]\right)^{2}+\left(y^{a}[n]-y^{b}[n]\right)^{2}+\left(z^{a}[n]-z^{b}[n]\right)^{2}}$ is the distance between nodes $a$ and $b$ in $n$th time slot. Further, $\alpha_{0}$ is the path-loss exponent, $\rho$ is a constant value depending on carrier frequency, which is commonly set as $\left[c /\left(4 \pi f_{c}\right)\right]^{2}$ with $c=3 \times 10^{8} \mathrm{~m} / \mathrm{s}$ and $f_{c}$ as the carrier frequency [35], and $g^{a, b}$ is the associated Rayleigh fading. Note that, because the legitimate nodes can communicate with each other, the UAV is assumed to have the perfect CDI for the legitimate nodes' channel gains, i.e. $g^{\mathrm{PT}, \mathrm{PR}}, g^{\mathrm{PT}, \mathrm{SR}}, g^{\mathrm{ST}, \mathrm{PR}}, g^{\mathrm{ST}, \mathrm{SR}}, g^{\mathrm{UAV}, \mathrm{PR}}$, and $g^{\mathrm{UAV}, \mathrm{SR}}$ experiencing frequency non-selective Rayleigh fading with zero mean and unit variance. Whereas, since Eve's location is not known, the UAV only has the partial CDI about Eve's channel gains, i.e. $g^{\text {ST,Eve }}$ and $g^{\mathrm{UAV} \text {,Eve }}$ with zero mean and unknown variances $\left(\sigma_{\mathrm{ST}, \mathrm{Eve}}^{2}\right.$ and $\left.\sigma_{\mathrm{UAV}, \text { Eve }}^{2}\right)$. The channel coefficients are independent and identically distributed (i.i.d.), and constant over each packet transmission [36]. Moreover, the

\footnotetext{
${ }^{1}$ In this paper our aim is to evaluate our proposed scheme from the fundamental perspectives, and the multiple antenna nodes will be considered in our future research.
}

UAV knows the noise variances at PR and SR, but does not know the noise variance at Eve.

\section{B. Signaling}

When the underlay strategy is adopted, the signals transmitted by PT and UAV interfere with secondary users. Hence, the received signal of $j$ th $(j \in\{1,2, \ldots, M\})$ channel in $n$th $(n \in\{1, \ldots, N\})$ time slot at $\mathrm{SR}$ can be expressed as

$$
\begin{aligned}
y_{j}^{\mathrm{SR}}[n]= & \sqrt{p^{\mathrm{ST}}} h^{\mathrm{ST}, \mathrm{SR}}[n] x_{j}^{\mathrm{ST}}[n]+\sqrt{p^{\mathrm{PT}}} h^{\mathrm{PT}, \mathrm{SR}}[n] x_{j}^{\mathrm{PT}}[n] \\
& +\sqrt{p^{\mathrm{UAV}}[n]} h^{\mathrm{UAV}, \mathrm{SR}}[n] x_{j}^{\mathrm{UAV}}[n]+n_{j}^{\mathrm{SR}}[n] .
\end{aligned}
$$

Above, $x_{j}^{\mathrm{ST}}[n], x_{j}^{\mathrm{PT}}[n]$, and $x_{j}^{\mathrm{UAV}}[n]$ respectively represent the $j$ th transmit symbol of the ST, PT, and UAV in the $n$th time slot. $p^{\mathrm{ST}}, p^{\mathrm{PT}}$ and $p^{\mathrm{UAV}}[n]$ respectively denote the transmit power at ST, PT and UAV in the $n$th time slot. Note that, $p^{\mathrm{ST}}$ and $p^{\mathrm{PT}}$ are considered as a priori and constant during the time period $T$ considered. Further, $h^{\mathrm{ST}, \mathrm{SR}}[n], h^{\mathrm{PT}, \mathrm{SR}}[n]$ and $h^{\mathrm{UAV}, \mathrm{SR}}[n]$ respectively denote the channel gains for the links of ST-SR, PT-SR and UAV-SR in the $n$th time slot. Last, $n_{j}^{\mathrm{SR}}[n]$ is the AWGN at SR with the variance $\sigma_{\mathrm{SR}}^{2}$.

Hence, the covert rate in $n$th $(n \stackrel{\in}{\in}\{1, \ldots, N\})$ time slot achieved by SR can be given by

$$
\begin{aligned}
R^{\mathrm{SR}, \mathrm{c}}[n] & =\frac{1}{M} \sum_{j=1}^{M} R_{j}^{\mathrm{SR}, \mathrm{c}}[n] \\
& =\log _{2}\left(1+\frac{p^{\mathrm{ST}}\left|h^{\mathrm{ST}, \mathrm{SR}}[n]\right|^{2}}{p^{\mathrm{PT}}\left|h^{\mathrm{PT}, \mathrm{SR}}[n]\right|^{2}+p^{\mathrm{UAV}}[n]\left|h^{\mathrm{UAV}, \mathrm{SR}}[n]\right|^{2}+\sigma_{\mathrm{SR}}^{2}}\right),
\end{aligned}
$$

where $M$ is the number of channel uses, and $R_{j}^{\mathrm{SR}, \mathrm{c}}[n]$ is the covert rate at SR for the $j$ th channel in the $n$th time slot. Upon requiring a certain quality-of-service $(\mathrm{QoS})$, i.e. $R_{\mathrm{PR}}^{\min }$, the achievable rate of $\mathrm{PR}$ in $n$th $(n \in\{1, \ldots, N\})$ time slot should satisfy that

$$
\begin{aligned}
R^{\mathrm{PR}}[n] & =\frac{1}{M} \sum_{j=1}^{M} R_{j}^{\mathrm{PR}}[n] \\
& =\log _{2}\left(1+\frac{p^{\mathrm{PT}}\left|h^{\mathrm{PT}, \mathrm{PR}}\right|^{2}}{p^{\mathrm{ST}}\left|h^{\mathrm{ST}, \mathrm{PR}}\right|^{2}+p^{\mathrm{UAV}}[n]\left|h^{\mathrm{UAV}, \mathrm{PR}}[n]\right|^{2}+\sigma_{\mathrm{PR}}^{2}}\right) \geq R_{\mathrm{PR}}^{\min },
\end{aligned}
$$

where $R_{j}^{\mathrm{PR}}[n]$ is the achievable rate of PR through $j$ th channel in $n$th time slot.

Further, in order to detect the presence of covert communication, Eve must distinguish whether ST sends information to $\mathrm{SR}$. Therefore, the received signal through $j$ th channel in $n$th time slot at Eve can be expressed as

$y_{j}^{\mathrm{Eve}}[n]= \begin{cases}\sqrt{p^{\mathrm{UAV}}[n]} h^{\mathrm{UAV}, \mathrm{Eve}}[n] x_{j}^{\mathrm{UAV}}[n]+n_{j}^{\mathrm{Eve}}[n], & H_{0}, \\ \sqrt{p^{\mathrm{UAV}}[n]} h^{\mathrm{UAV}, \mathrm{Eve}}[n] x_{j}^{\mathrm{UAV}}[n]+\sqrt{p^{\mathrm{ST}}} h^{\mathrm{ST}, \mathrm{Eve}} x_{j}^{\mathrm{ST}}[n]+n_{j}^{\mathrm{Eve}}[n], & H_{1},\end{cases}$

where $n_{j}^{\text {Eve }}[n]$ is the AWGN with variance $\sigma_{\text {Eve }}^{2}[n]$. Further, $H_{0}$ and $H_{1}$ respectively denote the hypothesis where ST has not or has transmitted covert message. When employing radiometer detection [17], Eve sets a pre-determined threshold $\tau[n]$, thus resulting in the following decision

$$
Y[n] \stackrel{D_{1}}{\underset{D_{0}}{\gtrless}} \tau[n],
$$


where $D_{0}$ and $D_{1}$ respectively correspond to a decision in favor of hypothesis $H_{0}$ and $H_{1}$, and $Y[n]$ represents the received signal power at Eve in $n$th $(n \in\{1, \ldots, N\})$ time slot, which can be written as

$$
\begin{aligned}
Y[n] & =\frac{1}{M} \sum_{j=1}^{M}\left|y_{j}^{\mathrm{Eve}}[n]\right|^{2} \\
& = \begin{cases}p^{\mathrm{UAV}}[n]\left|h^{\mathrm{UAV}, \mathrm{Eve}}[n]\right|^{2}+\sigma_{\text {Eve }}^{2}, & H_{0}, \\
p^{\mathrm{UAV}}[n]\left|h^{\mathrm{UAV}, \mathrm{Eve}}[n]\right|^{2}+p^{\mathrm{ST}}\left|h^{\mathrm{ST}, \mathrm{Eve}}\right|^{2}+\sigma_{\mathrm{Eve}}^{2}, & H_{1} .\end{cases}
\end{aligned}
$$

Hence, the probability of detection errors becomes

$$
\lambda[n]=P\left(H_{0}\right) P\left(D_{1} \mid H_{0}\right)[n]+P\left(H_{1}\right) P\left(D_{0} \mid H_{1}\right)[n],
$$

where $P\left(D_{1} \mid H_{0}\right)[n]$ and $P\left(D_{0} \mid H_{1}\right)[n]$ represent the probabilities of false alarm and missed detection in $n$th time slot, respectively. $P\left(H_{0}\right)$ (or $P\left(H_{1}\right)$ ) is the probability that ST does not (or does) transmit a covert message to SR. With the aid of the UAV jammer, we could assume that $P\left(H_{1}\right) \geq P\left(H_{0}\right)$.

\section{Problem Formulation}

In the considered system model, our objective is to maximize the covert rate as well as maximize the probability of detection errors at Eve by jointly optimizing the UAV's trajectory and jamming power. This multi-objective optimization problem can be described as

$$
\begin{aligned}
& \left(\mathcal{P}_{1}\right)_{\left\{x^{\mathrm{UAV}}[n], y^{\mathrm{UAV}}[n], z^{\mathrm{UAV}}[n], p^{\mathrm{UAV}}[n]\right\}} R^{\mathrm{SR}, \mathrm{c}}[n], \\
& \text { and } \quad\left(\mathcal{P}_{2}\right) \max _{\left\{x^{\mathrm{UAV}}[n], y^{\mathrm{UAV}}[n], z^{\mathrm{UAV}}[n], p^{\mathrm{UAV}}[n]\right\}} \lambda[n] \text {, } \\
& \text { s.t. } 0 \leq p^{\mathrm{UAV}}[n] \leq P_{\mathrm{UAV}}^{\max }, n \in\{1, \ldots, N\} \text {, }
\end{aligned}
$$$$
\text { (2), (6). }
$$

Above, $P_{\mathrm{UAV}}^{\max }$ denotes the maximum transmit power of UAV. Nevertheless, it is extremely difficult to find the optimal solution for the above problem. A range of facts result in this dilemma: First, the problem is multi-objective with high-dimensional variables, and is also non-convex. Second, to solve the problem, it requires the UAV to know the predetermined threshold used by Eve [37,38], which is obviously impractical. Third, to solve the problem, the instaneous channel information about $g^{\mathrm{ST}, \mathrm{Eve}}[n], g^{\mathrm{UAV}, \mathrm{Eve}}[n]$, and $\sigma_{\text {Eve }}^{2}[n]$, should be known by the UAV $[23,25]$, whereas in this work only the partial CDI of the channels are known.

On the other hand, Problem (11) can be seen as a game between a legitimate user (the UAV) and a warden (the Eve) in covert communication, and can be solved by a newly emerging machine learning method, namely GAN [31]. Basically, a GAN model consists of two modules, a generator as the legitimate user to generate a promising solution and a discriminator as the warden to discriminate the existence of covert message. In order to tackle the dynamic game, we design a modeldriven generative adversarial network (MD-GAN) optimization framework and propose a GAN based joint trajectory and power optimization (GAN-JTP) algorithm in the presence of unknown detection threshold and the imperfect CDI.

\section{Design of Model-Driven Generative AdVERSARial Network (MD-GAN)}

In this section, we analyze the multi-objective optimization problem, and design a MD-GAN.

\section{A. Problem Analysis}

In order to tackle the multi-objective optimization problem specified in (11), let us analyze the detection performance at the eavesdropper.

Lemma 1: The probabilities of false alarm and missed detection at Eve in $n$th $(n \in\{1, \ldots, N\})$ time slot are given by

$$
\begin{aligned}
& P\left(D_{1} \mid H_{0}\right)[n] \\
& = \begin{cases}e^{-\frac{\tau[n]-\sigma_{\mathrm{Eve}}^{2}}{2 b[n]},}, & \tau[n] \geq \sigma_{\mathrm{Eve}}^{2}, \\
1, & \tau[n]<\sigma_{\mathrm{Eve}}^{2},\end{cases}
\end{aligned}
$$

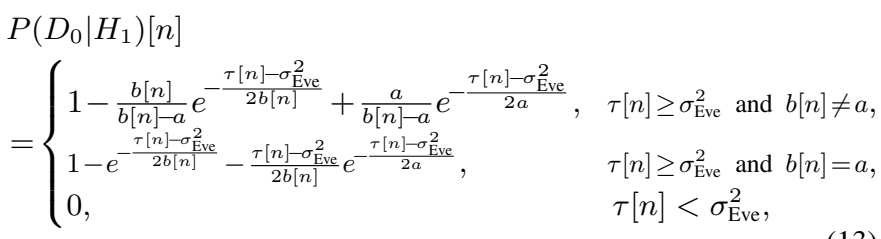

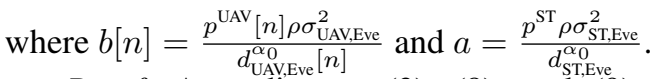

Proof: According to (3), (8), and (9), it is true that $P\left(D_{1} \mid H_{0}\right)[n]=P\left(\left|g^{\mathrm{UAV}, \mathrm{Eve}}[n]\right|^{2}>\frac{d_{\mathrm{UAV} V \mathrm{Eve}}^{\alpha}[n]\left(\tau[n]-\sigma_{\mathrm{Eve}}^{2}\right)}{\rho p^{\mathrm{VAV}}[n]}\right)$ and $P\left(D_{0} \mid H_{1}\right)[n]=P\left(\frac{\rho p^{\mathrm{UAV}}[n]}{d_{\text {UAV,Eve }}^{\alpha_{0}}}\left|g^{\mathrm{UAV}, \mathrm{Eve}}[n]\right|^{2}+\frac{\rho p^{\text {ST }}}{d_{\mathrm{ST}, \mathrm{Eve}}^{\alpha_{0}}}\left|g^{\mathrm{ST}, \mathrm{Eve}}[n]\right|^{2}<\right.$ $\left.\tau[n]-\sigma_{\text {Eve }}^{2}\right)$. Then, upon substituting the probability density functions (PDFs) $f_{\left|g^{\mathrm{UAV}, \text { Eve }}[n]\right|^{2}}(x)=\frac{1}{2 \sigma_{\text {UAV.Eve }}^{2}} e^{-\frac{x}{2 \sigma_{\text {UAVVVve }}^{2}}}$ and $f_{\left|g^{\text {ST,Eve }}[n]\right|^{2}}(x)=\frac{1}{2 \sigma_{\text {ST,Eve }}^{2}} e^{-\frac{x}{2 \sigma_{\text {ST,Eve }}^{2}}}$, we achieve the results in (12) and (13).

By submitting (12) and (13) into (10), we can obtain the probability of detection errors at Eve

$\lambda[n]=\left\{\begin{array}{l}k_{1} e^{-\frac{\tau[n]-\sigma_{\mathrm{Eve}}^{2}}{2 b \mid n]}}+k_{2} e^{-\frac{\tau[n]-\sigma_{\mathrm{Eve}}^{2}}{2 a}}+P\left(H_{1}\right), \tau[n] \geq \sigma_{\mathrm{Eve}}^{2} \text { and } b[n] \neq a, \\ P\left(H_{1}\right)+k_{3} e^{-\frac{\tau[n]-\sigma_{\mathrm{Eve}}^{2}}{2 a}}, \tau[n] \geq \sigma_{\mathrm{Eve}}^{2} \text { and } b[n]=a, \\ P\left(H_{0}\right), \tau[n]<\sigma_{\mathrm{Eve}}^{2}\end{array}\right.$

where $k_{1}=P\left(H_{0}\right)-\frac{b[n]}{b[n]-a} P\left(H_{1}\right), k_{2}=\frac{a}{b[n]-a} P\left(H_{1}\right)$, and $k_{3}=1-\left(2+\frac{\tau[n]-\sigma_{\mathrm{Eve}}^{2}}{2 a}\right) P\left(H_{1}\right)$.

In practice, it is impossible to know $\lambda[n]$ at the UAV since the threshold $\tau[n]$ adopted by Eve is unknown. However, as suggested in $[38,39]$, from the UAV's perspective, we are committed to find the optimal detection threshold $\tau[n]_{o p}$, which results in the minimal probability of detection errors $\lambda[n]_{\min }$ and will be used as initial value of network weight in the MD-GAN.

Theorem 1: The optimal detection threshold $\tau[n]_{o p}$ for Eve is

$$
\tau[n]_{o p}=\left\{\begin{array}{l}
\tau[n]^{*}, \tau[n] \geq \sigma_{\mathrm{Eve}}^{2} \text { and } b[n] \neq a \\
\frac{2 a P\left(H_{0}\right)}{P\left(H_{1}\right)}+\sigma_{\mathrm{Eve}}^{2}, \tau[n] \geq \sigma_{\mathrm{Eve}}^{2} \text { and } b[n]=a, \\
\text { nonexistent, } \tau[n]<\sigma_{\mathrm{Eve}}^{2}
\end{array}\right.
$$


where $\tau[n]^{*}=\frac{2 a b[n]}{a-b[n]} \ln \left[\left(\frac{a}{b[n]}-1\right) \frac{P\left(H_{0}\right)}{P\left(H_{1}\right)}+1\right]+\sigma_{\text {Eve }}^{2}$. The corresponding minimal probability of detection errors $\lambda[n]_{\min }$ at Eve is denoted by

$$
\lambda[n]_{\min }=\left\{\begin{array}{l}
\lambda[n]^{*}, \tau[n] \geq \sigma_{\mathrm{Eve}}^{2} \text { and } b[n] \neq a, \\
P\left(H_{1}\right)\left(1-e^{-\frac{P\left(H_{0}\right)}{P\left(H_{1}\right)}}\right), \tau[n] \geq \sigma_{\mathrm{Eve}}^{2} \text { and } b[n]=a, \\
P\left(H_{0}\right), \tau[n]<\sigma_{\mathrm{Eve}}^{2}
\end{array}\right.
$$

where $\lambda[n]^{*}=k_{1} e^{-\frac{\tau[n]^{*}-\sigma_{\text {Eve }}^{2}}{2 b[n]}}+k_{2} e^{-\frac{\tau[n]^{*}-\sigma_{\text {Eve }}^{2}}{2 a}}+P\left(H_{1}\right)$.

Proof: See Appendix A.

It is clearly observed in (16) that when $\tau[n] \geq \sigma_{\text {Eve }}^{2}$ and $b[n]=$ $a$, or $\tau[n]<\sigma_{\mathrm{Eve}}^{2}, \lambda[n]_{\min }$ is always a constant. By contrast, when $\tau[n] \geq \sigma_{\text {Eve }}^{2}$ and $b[n] \neq a$, the probability of detection errors should be minimized by transforming the multi-objective optimization problem (11) into

$$
\begin{array}{lll}
\left(\mathcal{P}_{3}\right) & \max & \left.R^{\mathrm{UAV}}[n], y^{\mathrm{UAV}}[n], z^{\mathrm{UAV}}[n], p^{\mathrm{UAV}}[n]\right\} \\
\text { s.t. } & \lambda[n]^{*} \geq 1-\epsilon, \\
& (2),(6),(11 \mathrm{c}) .
\end{array}
$$

Above constraint (17b) gives the covert requirement, where $\epsilon \in[0,1]$ is a predetermined value to specify the covert communication constraint $[15,29,39]$. Apparently, problem (17) is a non-convex problem. In the spirit of the approach proposed in $[23,40,41]$, we combine block coordinate descent (BCD) method and convex optimization to solve this complex highdimensional optimization problem. We decouple the original problem into UAV's trajectory subproblem and jamming power subproblem. In detail, we first optimize the UAV's jamming power while fixing UAV's trajectory, then we optimize the UAV's trajectory with a fixed jamming power. We repeat these two optimization subproblems until they converge and finally find the UAV's 3D trajectory and jamming power.

Theorem 2: When the UAV's 3D trajectory is fixed, the UAV's jamming power in $n$th $(n \in\{1, \ldots, N\})$ time slot can be given by

$$
p^{\mathrm{UAV}}[n]^{*}=\frac{a d_{\mathrm{UAV}, \mathrm{Eve}}^{\alpha_{0}}[n]}{\rho \sigma_{\mathrm{UAV}, \mathrm{Eve}}^{2}\left(1+M-\frac{P\left(H_{1}\right)}{P\left(H_{0}\right)}\right)},
$$

where $M=\frac{W\left\{\frac{P\left(H_{1}\right) \ln \left(\frac{\epsilon+P\left(H_{1}\right)-1}{P\left(H_{1}\right)}\right)}{P\left(H_{0}\right)}\left(\frac{\epsilon+P\left(H_{1}\right)-1}{P\left(H_{1}\right)}\right)^{\frac{P\left(H_{1}\right)}{P\left(H_{0}\right)}}\right\}}{\ln \left(\frac{\epsilon+P\left(H_{1}\right)-1}{P\left(H_{1}\right)}\right)}$, and $\mathrm{W}$ is the Lambert function [42].

Proof: See Appendix B.

Theorem 3: When the UAV's jamming power is fixed, the UAV's 3D trajectory $\left(x^{\mathrm{UAV}}[n]^{*}, y^{\mathrm{UAV}}[n]^{*}, z^{\mathrm{UAV}}[n]^{*}\right)$ in $n$th time slot is a tractable solution of the following approximated optimization problem

$$
\begin{array}{lc}
\left(\mathcal{P}_{4}\right) & \max _{\left\{x^{\mathrm{UAV}}[n], y^{\mathrm{UAV}}[n], z^{\mathrm{UAV}}[n]\right\}} \\
\text { s.t. } & (2), \\
& \left.d_{\mathrm{UAV}, \mathrm{Eve}}[n]^{2} \leq R_{\mathrm{Eve}, \mathrm{SR}}[n]^{2}\right]^{\frac{2}{\alpha_{0}}}, \\
& \left(x^{\mathrm{UAV}}[n], y^{\mathrm{UAV}}[n], z^{\mathrm{UAV}}[n]\right) \in S_{u},
\end{array}
$$

where $R_{\mathrm{Eve}}[n]=\frac{p^{\mathrm{UAV}}[n] \rho \sigma_{\mathrm{UAV}, \mathrm{Eve}}^{2}\left(1+M-\frac{P\left(H_{1}\right)}{P\left(H_{0}\right)}\right)}{a}$, and $S_{u}$ is the possible region which is on or outside the sphere centered at the
PR with radius $R_{\mathrm{PR}}=\frac{\rho p^{\mathrm{UAV}}[n]}{\frac{p^{\mathrm{PT}}\left|h_{\mathrm{PT}, \mathrm{PR}}\right|^{2}}{2^{R_{\mathrm{PR}}^{\min }}-1}-p^{\mathrm{ST}}\left|h^{\mathrm{ST}, \mathrm{PR}}\right|^{2}-\sigma_{\mathrm{PR}}^{2}}$, and derived from the tangent plane approximation as shown in [23].

Proof: See Appendix C.

On the next step, we design a MD-GAN optimization framework which mimics a game between the UAV and Eve by iteratively solving (18), (19), and (14).

\section{B. MD-GAN Optimization Framework}

As shown in Fig. 2, the designed MD-GAN optimization framework consists of two modules: 1) a generator, G, generating the UAV's trajectory and transmit power; 2) a discriminator, D, discriminating the existence of covert message. From the UAV's perspective, the MD-GAN transforms the whole optimization process into an interaction game between the generator (UAV) and the discriminator (Eve).

1) Generator: First, according to problems (18) and (19), the generator selects the locations $Q=$ $\left\{x^{\mathrm{ST}}, y^{\mathrm{ST}}, z^{\mathrm{ST}}, x^{\mathrm{SR}}, y^{\mathrm{SR}}, z^{\mathrm{SR}}, x^{\mathrm{PT}}, y^{\mathrm{PT}}, z^{\mathrm{PT}}, x^{\mathrm{PR}}, y^{\mathrm{PR}}, z^{\mathrm{PR}}, \hat{x}^{\mathrm{Eve}}\right.$, $\left.\hat{y}^{\mathrm{Eve}}, \hat{z}^{\mathrm{Eve}}\right\}$ and the current environment status $I=$ $\left\{P\left(H_{1}\right), P\left(H_{0}\right), p^{\mathrm{ST}}, p^{\mathrm{PT}}, f_{c}, \alpha_{0}, \varepsilon_{E v e}^{2}, V_{\max }, P_{\mathrm{UAV}}^{\max }, H_{\min }, \delta, \epsilon\right.$, $\left.R_{\mathrm{PR}}^{\min }, \sigma_{\mathrm{PR}}^{2}, \sigma_{\mathrm{SR}}^{2}\right\}$ as its inputs. Then, the generator produces the optimized trajectory and transmit power for UAV by solving problems (18) and (19). In particular, if the problems (18) and (19) converge after $L$ iterations, the generator contains $L-1$ power generation layers, $L-1$ trajectory generation layers, one power output layer, and one trajectory output layer.

Furthermore, in each power generation layer and power output layer, there is only one neuron $p^{\mathrm{UAV}}[n]^{*}$, containing $N$ data. The non-linear transform of neuron $p^{\mathrm{UAV}}[n]^{*}$, i.e. activation function, is designed according to (18). Similarly, in each trajectory generation layer and trajectory output layer, there are three types of neurons, i.e., $x^{\mathrm{UAV}}[n]^{*}, y^{\mathrm{UAV}}[n]^{*}$, and $z^{\mathrm{UAV}}[n]^{*}$, each of them contains $N$ data. The non-linear transform of neurons $x^{\mathrm{UAV}}[n]^{*}, y^{\mathrm{UAV}}[n]^{*}$, and $z^{\mathrm{UAV}}[n]^{*}$ is obtained by solving (19). Note that, in each of the neurons of the generator, the channel distribution variances $\sigma_{\mathrm{ST} \text {,Eve }}^{2}$ and $\sigma_{\mathrm{UAV} \text {,Eve }}^{2}$ are the parameters to be learned.

2) Discriminator: The discriminator performs the operation derived from (14). Hence, the discriminator contains one decision output layer, where the UAV's trajectory and transmit power solution generated by $\mathrm{G}$, and the partial environment status, i.e., $E=$ $\left\{x^{\mathrm{ST}}, y^{\mathrm{ST}}, z^{\mathrm{ST}}, \hat{x}^{\mathrm{Eve}}, \hat{y}^{\mathrm{Eve}}, \hat{z}^{\mathrm{Eve}}, P\left(H_{1}\right), P\left(H_{0}\right), p^{\mathrm{ST}}, f_{c}, \alpha_{0}, \varepsilon_{\mathrm{Eve}}^{2}\right\}$, are the inputs. Further, there is only one neuron $\lambda[n]$ in this layer, which contains $N$ data. The non-linear transform of neuron $\lambda[n]$ is designed according to (14). In addition, the channel distribution variances $\sigma_{\text {ST,Eve }}^{2}$ and $\sigma_{\mathrm{UAV}, \mathrm{Eve}}^{2}$, the AWGN variance $\sigma_{\text {Eve }}^{2}$, and the detection threshold $\tau[n]$ are the parameters to be learned.

\section{GAN BASED JOINT TRAJECTORY AND POWER OPTIMIZATION (GAN-JTP) ALGORITHM}

In this section, we design a GAN based joint trajectory and power optimization algorithm, namely GAN-JTP, which provides the optimized UAV's 3D trajectory and transmit power. 


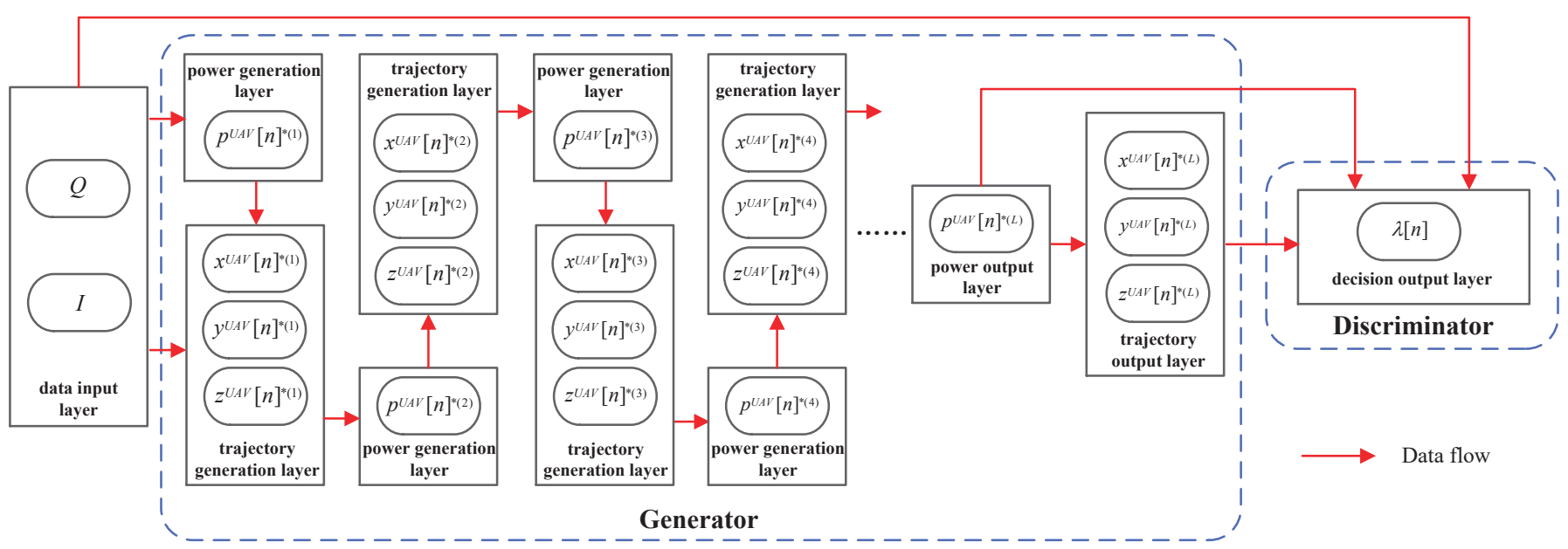

Fig. 2. Architecture of the MD-GAN optimization framework.

\section{A. Design objective of GAN-JTP algorithm}

The GAN-JTP algorithm is designed to optimize the generator and discriminator of the MD-GAN framework that are both operated by the UAV and alternatively trained in a competitive manner. From the UAV's perspective, the MD-GAN can make the generator to produce the UAV's trajectory and transmit power solution, while the discriminator imitating Eve to discriminate the existence of covert message. Note that, our goal is to achieve the covert communication for the ST, which aims to design an effective generator that can generate the joint solution of trajectory and transmit power for the UAV to help ST to deceive the discriminator playing as the Eve. As shown in Fig. 3, the GAN-JTP consists of two stages: the network learning stage and the network training stage. During the network learning stage, as described in Section III-B, the generator selects the locations $Q$ and current environment status $I$ as the inputs to optimize the UAV's 3D trajectory and transmit power; While, the discriminator uses the partial environment status $E$, the UAV's 3D trajectory and transmit power solution (generated by the generator) as the inputs to compute the probability of detection errors $\lambda[n]$ at Eve. Then, during the network training stage, the loss functions $L_{\mathrm{G}}$ and $L_{\mathrm{D}}$ are constructed to train the generator and the discriminator, where the channel distribution variances $\sigma_{\mathrm{ST}, \mathrm{Eve}}^{2}$ and $\sigma_{\mathrm{UAV}, \mathrm{Eve}}^{2}$, the AWGN variance $\sigma_{\mathrm{Eve}}^{2}$, and the detection threshold $\tau[n]$ are learned and updated from the view of the UAV.

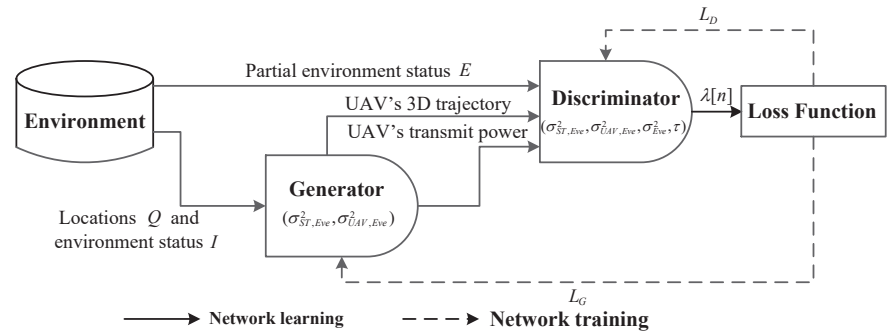

Fig. 3. The structure of GAN-JTP algorithm.
1) Network learning stage of GAN-JTP algorithm: This stage is a forward propagation process, and its principles are summarized in Algorithm 1. First, let us update the current state, initialize the network weight sets and UAV's 3D trajectory, and set key parameters, as shown in steps 1 to 3 . Second, the UAV's 3D trajectory and transmit power are obtained along with the data flow of the generator, as shown in steps 4 to 9 . Specifically, to analyze the covert performance during the time period $T$ considered, we set the average values $\overline{R^{\mathrm{SR}, \mathrm{c}}}=\frac{1}{N} \sum_{n=1}^{N} R^{\mathrm{SR}, \mathrm{c}}(n)$ and $\bar{\lambda}=\frac{1}{N} \sum_{n=1}^{N} \lambda(n)$. When the convergence condition is satisfied, the joint optimization solution is acquired. Finally, upon substituting the results of generator, the probability of detection errors $\lambda[n]$ is computed along with the data flow of the discriminator, as shown in step 10. Note that, we initialize the network weights based on the characteristics of covert communication in practical UAV scenarios. As suggested by [29], the initial values of UAV's 3D trajectory $\left(x^{\mathrm{UAV}}[n]^{*(0)}, y^{\mathrm{UAV}}[n]^{*(0)}, z^{\mathrm{UAV}}[n]^{*(0)}\right)_{n=1}^{N}$ can be obtained as follows. The UAV first flies from the takeoff location to Eve via the shortest path and at the maximum speed, then it hovers above the Eve's estimated location as long as possible, finally it flies to the landing location via the shortest path and at the maximum speed.

\begin{tabular}{|l|}
\hline Algorithm 1: Network Learning Stage of GAN-JTP Algorithm \\
\hline 1:Update current location information $Q$ and environment status $I ;$ \\
2:Initialize the network weight sets $\theta_{1}=\left\{\sigma_{\mathrm{ST}, \mathrm{Eve}}^{2(l)}, \sigma_{\mathrm{UAV}, \text { Eve }}^{2(l)}\right\}_{l=1}^{L}$ of the \\
generator, $\theta_{2}=\left\{\sigma_{\mathrm{ST}, \mathrm{Eve}}^{2}, \sigma_{\mathrm{UAV}, \mathrm{Eve}}^{2}, \sigma_{\mathrm{Eve}}^{2}, \tau[n]\right\}_{n=1}^{N}$ of the discriminator, \\
and $\left(x^{\mathrm{UAV}}[n]^{*(0)}, y^{\mathrm{UAV}}[n]^{*(0)}, z^{\mathrm{UAV}}[n]^{*(0)}\right)_{n=1}^{N}$; \\
3:Set threshold $\xi$ and maximum iteration number $L_{\text {max }}$; \\
4:For $l=1, \ldots, L_{\max }$ \\
5:Calculate $\left\{p^{\mathrm{UAV}}[n]^{*(l)}, x^{\mathrm{UAV}}[n]^{*(l)}, y^{\mathrm{UAV}}[n]^{*(l)}, z^{\mathrm{UAV}}[n]^{*(l)}\right\}_{n=1}^{N}$ \\
along with the data flow of the generator; \\
6:If $\left|\frac{R^{\mathrm{SR}, \mathrm{c}}(l)}{R^{\mathrm{SR}, \mathrm{c}}}(l-1)\right|<\xi$ and $\left|\bar{\lambda}^{(l)}-\bar{\lambda}^{(l-1)}\right|<\xi$ \\
7:Output $\left\{p^{\mathrm{UAV}}[n]^{*}(l), x^{\mathrm{UAV}}[n]^{*(l)}, y^{\mathrm{UAV}}[n]^{*(l)}, z^{\mathrm{UAV}}[n]^{*(l)}\right\}_{n=1}^{N}$ as \\
the solution $\left\{p^{\mathrm{UAV}}[n]^{*}, x^{\mathrm{UAV}}[n]^{*}, y^{\mathrm{UAV}}[n]^{*}, z^{\mathrm{UAV}}[n]^{*}\right\}_{n=1}^{N} ;$ \\
8:End If \\
9:End For \\
10:Output $\lambda[n](n \in\{1, \ldots, N\})$ along with the data flow of the \\
discriminator.
\end{tabular}


2) Network training stage of GAN-JTP algorithm: The network training stage is a backward propagation process. According to [43], the training procedure is a two player min-max game which can be expressed by

$$
\min _{D} \max _{G} V(G, D)=R^{\mathrm{SR}, \mathrm{c}}[n]+D(G(s)[n]),
$$

where $R^{\mathrm{SR}, \mathrm{c}}[n]$ represents the covert rate at SR. Further, $s=\{Q, I\}$ is the current state observed from the network environment, and $D(G(s)[n])$ denotes the probability of detection errors in $n$th time slot where $G(s)[n]=$ $\left\{p^{\mathrm{UAV}}[n]^{*}, x^{\mathrm{UAV}}[n]^{*}, y^{\mathrm{UAV}}[n]^{*}, z^{\mathrm{UAV}}[n]^{*}\right\}$. Note that, according to problem (11), the training objective of generator (UAV) aims at maximizing the probability of detection errors $D(G(s)[n])$, and at maximizing the covert rate $R^{\mathrm{SR}, \mathrm{c}}[n]$. By contrast, the objective of discriminator (Eve) is to minimize the probability of detection errors $D(G(s)[n])$, and hence the existence of covert message could be discriminated.

According to the proposals in [44], we adopt gradien$\mathrm{t}$ back-propagation to iteratively train the weights of generator and discriminator. In order to avoid over-fit, we alternate between $k_{d}$ steps of optimizing the discriminator and one step of optimizing the generator. Hence, the weight sets $\theta_{1}=\left\{\sigma_{\mathrm{ST}, \mathrm{Eve}}^{2(l)}, \sigma_{\mathrm{UAV}, \mathrm{Eve}}^{2(l)}\right\}_{l=1}^{L}$ of generator and $\theta_{2}=$ $\left\{\sigma_{\text {ST,Eve }}^{2}, \sigma_{\text {UAV,Eve }}^{2}, \sigma_{\text {Eve }}^{2}, \tau[n]\right\}_{n=1}^{N}$ of discriminator are respectively updated along the gradient ascending and descending directions of the following loss functions

$$
\begin{aligned}
& L_{\mathrm{G}}=\nabla \theta_{1} \frac{1}{N} \sum_{n=1}^{N}\left(R^{\mathrm{SR}, \mathrm{c}}[n]+D(G(s))[n]\right), \\
& L_{\mathrm{D}}=\nabla \theta_{2} \frac{1}{N} \sum_{n=1}^{N}(D(G(s))[n]) .
\end{aligned}
$$

In the next, let us briefly introduce the gradient computation with the aid of a typical stage.

Discriminator: The discriminator has one node $\lambda[n]$, which has four inputs and one output, as shown in Fig. 4 (a). The gradients of loss function $L_{\mathrm{D}}$ in (22) with regard to the learned weights $\sigma_{\mathrm{ST} \text {,Eve }}^{2}, \sigma_{\mathrm{UAV}, \mathrm{Eve}}^{2}, \sigma_{\mathrm{Eve}}^{2}$, and $\tau[n]$ can be calculated as

$$
\left\{\begin{array}{l}
\frac{\partial L_{\mathrm{D}}}{\partial \sigma_{\text {ST,Eve }}^{2}}=\frac{\partial L_{\mathrm{D}}}{\partial \lambda[n]} \frac{\partial \lambda[n]}{\partial \sigma_{\text {ST,Eve }}^{2}} \\
\frac{\partial L_{\mathrm{D}}}{\partial \sigma_{\mathrm{UAV}, \mathrm{Eve}}^{2}}=\frac{\partial L_{\mathrm{D}}}{\partial \lambda[n]} \frac{\partial \lambda[n]}{\partial \sigma_{\mathrm{UAV}, \mathrm{Eve}}^{2}} \\
\frac{\partial L_{\mathrm{D}}}{\partial \sigma_{\text {Eve }}^{2}}=\frac{\partial L_{\mathrm{D}}}{\partial \lambda[n]} \frac{\partial \lambda[n]}{\partial \sigma_{\text {Eve }}^{2}} \\
\frac{\partial L_{\mathrm{D}}}{\partial \tau[n]}=\frac{\partial L_{\mathrm{D}}}{\partial \lambda[n]} \frac{\partial \lambda[n]}{\partial \tau[n]}
\end{array}\right.
$$

where $\frac{\partial L_{\mathrm{D}}}{\partial \lambda[n]}=1$ and can be derived from (22). Meanwhile, $\frac{\partial \lambda[n]}{\partial \sigma_{\text {STEve }}^{2}}, \frac{\partial \lambda[n]}{\partial \sigma_{\text {UAV,Eve }}^{2}}, \frac{\partial \lambda[n]}{\partial \sigma_{\text {Eve }}^{2}}$, and $\frac{\partial \lambda[n]}{\partial \tau[n]}$ can be calculated according to (14).

For the loss function $L_{\mathrm{D}}$, the weight set $\theta_{2}$ can be updated along the descending direction of the gradient, described as

$$
\left\{\begin{array}{l}
\sigma_{\mathrm{ST}, \mathrm{Eve}}^{2} \leftarrow \sigma_{\mathrm{ST}, \mathrm{Eve}}^{2}-\lambda_{\sigma_{\mathrm{ST}, \mathrm{Eve}}^{2}} \frac{\partial L_{\mathrm{D}}}{\partial \sigma_{\mathrm{ST}, \mathrm{Eve}}^{2}} \\
\sigma_{\mathrm{UAV}, \mathrm{Eve}}^{2} \leftarrow \sigma_{\mathrm{UAV}, \mathrm{Eve}}^{2}-\lambda_{\sigma_{\mathrm{UAV}, \mathrm{Eve}}^{2}} \frac{\partial L_{\mathrm{D}}}{\partial \sigma_{\mathrm{UAV}, \mathrm{Eve}}^{2}} \\
\sigma_{\mathrm{Eve}}^{2} \leftarrow \sigma_{\mathrm{Eve}}^{2}-\lambda_{\sigma_{\mathrm{Eve}}^{2}} \frac{\partial L_{\mathrm{D}}}{\partial \sigma_{\mathrm{Eve}}^{2}} \\
\tau[n] \leftarrow \tau[n]-\lambda_{\tau[n] \frac{\partial L_{\mathrm{D}}}{\partial \tau[n]}}
\end{array},\right.
$$

where $\lambda_{\sigma_{\text {STEve }}^{2}}, \lambda_{\sigma_{\text {UAVEve }}^{2}}, \lambda_{\sigma_{\text {Eve }}^{2}}$ and $\lambda_{\tau[n]}$ are adaptive learning rates, and are updated according to the function $L_{\mathrm{D}}$. For example, the adaptive learning rate $\lambda_{\sigma_{\text {STEve }}^{2}}$ in the $t+1$-st network training stage is given by

$$
\lambda_{\sigma_{\mathrm{ST}, \mathrm{Eve}}^{2}}(t+1)= \begin{cases}1.2 \lambda_{\sigma_{\mathrm{ST}, \mathrm{Eve}}^{2}}(t) & L_{\mathrm{D}}(t)<L_{\mathrm{D}}(t-1), \\ 0.7 \lambda_{\sigma_{\mathrm{ST}, \mathrm{Eve}}^{2}}(t) & L_{\mathrm{D}}(t)>1.04 L_{\mathrm{D}}(t-1), \\ \lambda_{\sigma_{\mathrm{ST}, \mathrm{Eve}}^{2}(t)} & \text { otherwise. }\end{cases}
$$

Generator: The generator has eight nodes. As shown in Fig. 4 (c), the inputs of the node $p^{\mathrm{UAV}}[n]^{*(l)}$ are $x^{\mathrm{UAV}}[n]^{*(l-1)}$, $y^{\mathrm{UAV}}[n]^{*(l-1)}$, and $z^{\mathrm{UAV}}[n]^{*(l-1)}$, and its output is $p^{\mathrm{UAV}}[n]^{*(l)}$. The gradients of loss function with regard to $\sigma_{\text {ST,Eve }}^{2}$ and $\sigma_{\text {UAV,Eve }}^{2}$ are given by

$$
\left\{\begin{array}{l}
\frac{\partial L_{\mathrm{G}}}{\partial \sigma_{\mathrm{STEve}}^{2(l)}}=\frac{\partial L_{\mathrm{G}}}{\partial p^{\mathrm{UAV}}[n]^{*(l)}} \frac{\partial p^{\mathrm{UAV}}[n]^{*(l)}}{\partial \sigma_{\mathrm{STE}}^{2(l)}} \\
\frac{\partial L_{\mathrm{G}}}{\partial \sigma_{\mathrm{UAV}, \mathrm{Eve}}^{2(l)}}=\frac{\partial L_{\mathrm{G}}}{\partial p^{\mathrm{UAV}}[n]^{*(l)}} \frac{\partial p^{\mathrm{UAV}}[n]^{*(l)}}{\partial \sigma_{\mathrm{UAV}, \mathrm{Eve}}^{2(l)}}
\end{array}\right.
$$

where $\frac{\partial p^{\mathrm{UAV}}[n]^{*(l)}}{\partial \sigma_{\text {ST,Eve }}^{2(l)}}$ and $\frac{\partial p^{\mathrm{UAV}}[n]^{*(l)}}{\partial \sigma_{\mathrm{UAV}, \mathrm{Eve}}^{2(l)}}$ can be obtained from (18). Further, $\frac{\partial L_{G}}{\partial p^{\mathrm{UAV}}[n]^{*(l)}}$ can be expressed as

$$
\begin{gathered}
\frac{\partial L_{\mathrm{G}}}{\partial p^{\mathrm{UAV}}[n]^{*(l)}}=\frac{\partial L_{\mathrm{G}}}{\partial x^{\mathrm{UAV}}[n]^{*(l)}} \frac{\partial x^{\mathrm{UAV}}[n]^{*(l)}}{\partial p^{\mathrm{UAV}}[n]^{*(l)}} \\
+\frac{\partial L_{\mathrm{G}}}{\partial y^{\mathrm{UAV}}[n]^{*(l)}} \frac{\partial y^{\mathrm{UAV}}[n]^{*(l)}}{\partial p^{\mathrm{UAV}}[n]^{*(l)}}+\frac{\partial L_{\mathrm{G}}}{\partial z^{\mathrm{UAV}}[n]^{*(l)}} \frac{\partial z^{\mathrm{UAV}}[n]^{*(l)}}{\partial p^{\mathrm{UAV}}[n]^{*(l)}}
\end{gathered}
$$

In order to maximize the loss function $L_{\mathrm{G}}$, the weight set $\theta_{1}$ can be updated along the ascending direction of the gradient, expressed as

$$
\left\{\begin{array}{l}
\sigma_{\mathrm{ST}, \mathrm{Eve}}^{2} \leftarrow \sigma_{\mathrm{ST}, \mathrm{Eve}}^{2}+\lambda_{\sigma_{\mathrm{ST}, \mathrm{Eve}}^{2}} \frac{\partial L_{\mathrm{G}}}{\partial \sigma_{\mathrm{ST}, \mathrm{Eve}}^{2}} \\
\sigma_{\mathrm{UAV}, \mathrm{Eve}}^{2} \leftarrow \sigma_{\mathrm{UAV}, \mathrm{Eve}}^{2}+\lambda_{\sigma_{\mathrm{UAV}, \mathrm{Gve}}^{2}} \frac{\partial L_{\mathrm{UAV}, \mathrm{Eve}}}{\partial \sigma^{2}}
\end{array},\right.
$$

where $\lambda_{\sigma_{\text {STEve }}^{2}}$ and $\lambda_{\sigma_{\text {UAVEve }}^{2}}$ are adaptive learning rates, and are updated by loss function $L_{\mathrm{G}}$. Furthermore, the adaptive learning rate $\lambda_{\sigma_{\text {STEVe }}^{2}}$ in the $t+1$-st network training stage can be updated by

$$
\lambda_{\sigma_{\mathrm{ST}, \mathrm{Eve}}^{2}}(t+1)= \begin{cases}1.2 \lambda_{\sigma_{\mathrm{ST}, \mathrm{Eve}}^{2}}(t) & L_{\mathrm{G}}(t)>L_{\mathrm{G}}(t-1), \\ 0.7 \lambda_{\sigma_{\mathrm{ST}, \mathrm{Eve}}^{2}}(t) & 1.04 L_{\mathrm{G}}(t)<L_{\mathrm{G}}(t-1), \\ \lambda_{\sigma_{\mathrm{ST}, \mathrm{Eve}}^{2}}(t) & \text { otherwise }\end{cases}
$$

Note that, in the other nodes, the gradient of loss function $L_{\mathrm{G}}$ and the update approach with regard to $\sigma_{\mathrm{ST} \text {,Eve }}^{2}$ and $\sigma_{\mathrm{UAV} \text {,Eve }}^{2}$ are similar to those applied to the node $p^{\mathrm{UAV}}[n]^{*(l)}$.

The detailed procedures of the network training stage are summarized in Algorithm 2. First, the key parameters are set, as shown in step 1 . Second, the loss function $L_{\mathrm{D}}$ and $L_{\mathrm{G}}$ are calculated upon the optimization solution generated in Algorithm 1, as shown in steps 2 to 3. Finally, when the convergence conditions are not satisfied, we repeat optimizing the discriminator and generator, and re-execute the Algorithm 1 ; Otherwise, the promising solution is obtained, as shown in steps 4 to 14 .

As suggested by $[30,45]$, the computational complexity of GAN-JTP algorithm can be reflected by the total number of optimization variables required to be calculated. Specifically, during the network learning stage, the transmit power neuron 


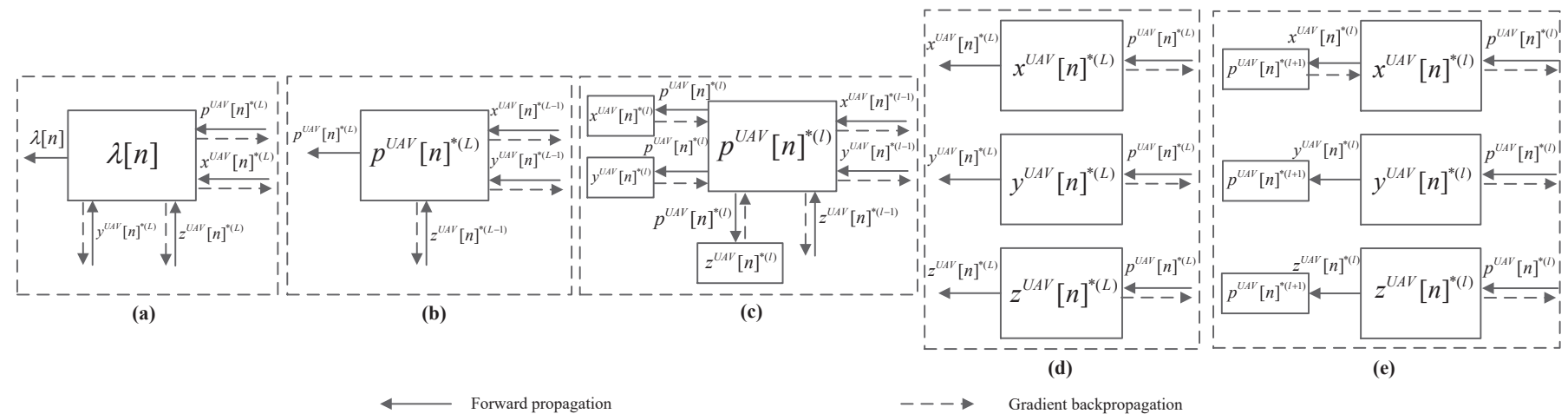

Fig. 4. The data flow and gradient backpropagation direction over each node.

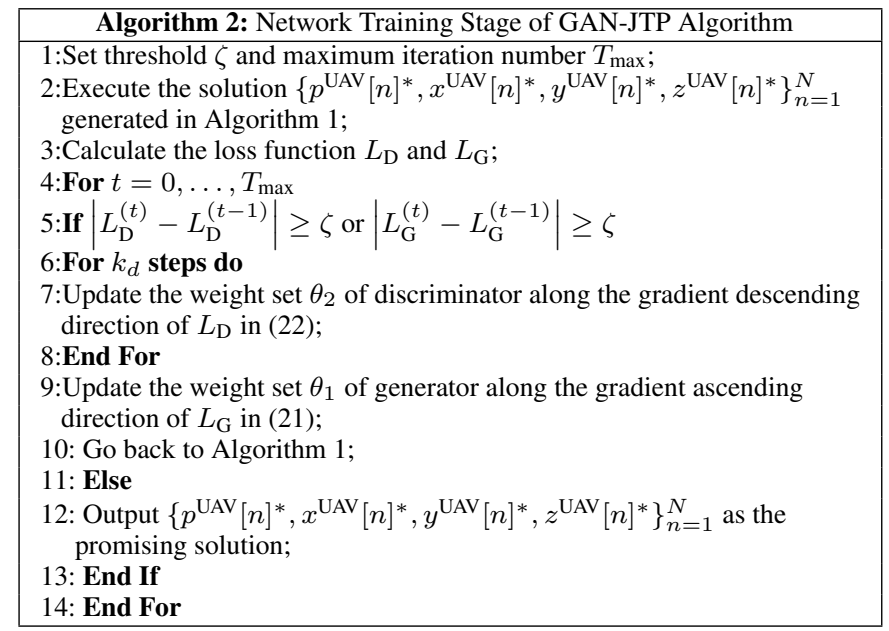

$p^{\mathrm{UAV}}[n]^{*}$ and trajectory neurons, i.e., $x^{\mathrm{UAV}}[n]^{*}, y^{\mathrm{UAV}}[n]^{*}$, and $z^{\mathrm{UAV}}[n]^{*}$, are calculated along with the data flow of the generator, while the decision neuron $\lambda[n]$ is computed along with the data flow of the discriminator. In the generator, if we assume that the data flow has $l$ iterations, each of the neurons $p^{\mathrm{UAV}}[n]^{*}$, $x^{\mathrm{UAV}}[n]^{*}, y^{\mathrm{UAV}}[n]^{*}$, and $z^{\mathrm{UAV}}[n]^{*}$ needs to be calculated by $l * N$ times. In the discriminator, the neuron $\lambda[n]$ needs to be calculated by $N$ times. Therefore, the computational complexity of the network learning stage is $\mathcal{O}(l * N)$. During the network training stage, the network weights in both generator and discriminator are updated by adopting the gradient backpropagation of the loss function. In the generator, the learned weights $\sigma_{\mathrm{ST}, \text { Eve }}^{2}$ and $\sigma_{\mathrm{UAV}, \text { Eve }}^{2}$ need to be updated by $4 * l * N$ times. In the discriminator, each of the learned weights $\sigma_{\text {STEve }}^{2}$, $\sigma_{\mathrm{UAV}, \mathrm{Eve}}^{2}, \sigma_{\mathrm{Eve}}^{2}$, and $\tau$ needs to be updated by $N$ times. Hence, the computational complexity of the network training stage becomes $\mathcal{O}(l * N)$. When assuming cyclic network learning and network training stages are executed $t$ times, the ultimate computational complexity of the proposed GAN-JTP algorithm is given by $\mathcal{O}(t * l * N)$.

\section{Simulation Results}

In this section, we consider a UAV-enabled cooperative jamming CRN with PT, PR, ST, and SR, whose 3D coordinates are given by $(100,100,0),(600,100,0),(250,1000,0)$, and $(750,1000,0)$. The UAV is assumed to fly from the initial location $(100,1000,100)$ towards the final location
$(600,1000,100)$ with the maximum speed $V_{\max }=5 \mathrm{~m} / \mathrm{s}$ and the maximum transmit power $P_{\mathrm{UAV}}^{\max }=20 \mathrm{dBm}$. Other simulation parameters are set as $p^{\mathrm{PT}}=35 \mathrm{dBm}, p^{\mathrm{ST}}=20 \mathrm{dBm}, \epsilon=0.1, \delta=1 \mathrm{~s}$, $H_{\min }=30 \mathrm{~m}, R_{\mathrm{PR}}^{\min }=1 \mathrm{bit} / \mathrm{s} / \mathrm{Hz}, P\left(H_{1}\right)=0.92, P\left(H_{0}\right)=0.08$, $\alpha_{0}=2, \sigma_{\mathrm{PR}}^{2}=\sigma_{\mathrm{SR}}^{2}=-109 \mathrm{dBm}, f_{c}=30 \mathrm{MHz}, \varepsilon_{\mathrm{Eve}}^{2}=25$. Specially, for GAN, we set the hyper-parameters $\xi=\zeta=0.0001$, $L_{\max }=T_{\max }=1000$. Furthermore, we initialize all the learning rates by 0.01 , the variances $\sigma_{\mathrm{ST} \text {,Eve }}^{2}$ and $\sigma_{\mathrm{UAV}, \mathrm{Eve}}^{2}$ by random variables with zero mean and unit variance, the noise variance $\sigma_{\text {Eve }}^{2}$ by white Gaussian noise, and $\tau[n]$ by the optimal detection threshold derived from (15). In the simulation, we consider the worst case scenario that Eve can perform the detection with the optimal $\tau[n]_{o p}$ (to minimize $\lambda[n]$ ).

\section{A. Comparison}

In this section, we provide numerical results to compare the performance of the GAN-JTP algorithm with the single trajectory and transmit power optimization algorithm (STP) [29] and BCD algorithm [23]. The STP algorithm fixes the UAV's 3D trajectory, and only optimizes UAV's transmit power by convex optimization. The BCD algorithm repeats two optimization subproblems shown as (18) and (19) until they converge and finally finds the UAV's 3D trajectory and jamming power. It is worth to emphasize that, both the STP algorithm and BCD algorithm have to require perfect CSI and Eve's detection threshold, in contrast, the proposed GAN-JTP algorithm only demands imperfect knowledge of CDI, and is blind to the detection threshold used by Eve.

Fig. 5 compares average covert rate $\overline{R^{\mathrm{SR}, \mathrm{c}}}$ and average probability of detection errors $\bar{\lambda}$. In this figure, we first observe that $R^{\mathrm{SR}, \mathrm{c}}$ and $\bar{\lambda}$ achieved by these three algorithms increase as $T$ increases. It shows that, the increase of $T$ allows the UAV to hover around Eve for a longer time, thus makes the covert transmission become more difficult to be detected. Further, $\overline{R^{\mathrm{SR}, \mathrm{c}}}$ and $\bar{\lambda}$ decrease as $\varepsilon_{\mathrm{Eve}}^{2}$ increases, since a larger $\varepsilon_{\mathrm{Eve}}^{2}$ makes the covertness constraints more difficult to be satisfied. This observation also shows that the Eve's location uncertainty level has an explicit impact on the covert rate performance. In addition, for $T=100 s, \overline{R^{\mathrm{SR}, \mathrm{c}}}$ derived by the GAN-JTP algorithm is slightly larger than that achieved by the STP algorithm and BCD algorithm, where the gain is obtained at the cost of sacrificing $\bar{\lambda}$. This is because that, $T=100 \mathrm{~s}$ is the minimum time required by the UAV to fly from the 
initial location to the final location under the maximum speed constraint. Different with the STP algorithm and BCD algorithm having perfect CSI, the GAN-JTP algorithm is operated based on the CDI with certain errors, which further complicates the optimization of UAV's transmit power. However, with the increase of $T$, the GAN-JTP algorithm and BCD algorithm are distinctly superior to the STP algorithm in term of $\overline{R^{\mathrm{SR}, \mathrm{c}}}$ and $\bar{\lambda}$. This observation shows that the joint optimization of the UAV's trajectory and transmit power is necessary for improving the covert communication performance. Furthermore, $\bar{\lambda}$ of the GAN-JTP is reduced by $0.15 \%$ with $0.47 \%$ increase of $\overline{R^{\mathrm{SR}, \mathrm{c}}}$ compared to the BCD algorithm. This is because in the GANJTP, the UAV is over-optimistic about the detection ability of Eve, thereby increasing the detection risk. Whereas, the BCD algorithm requires the UAV to know perfect CSI and Eve's detection threshold. Hence, the above observations demonstrate that the GAN-JTP algorithm can attain near-optimal solutions of UAV's trajectory and transmit power, and is more practical.

\section{B. Effects of training steps}

Fig. 6 evaluates average covert rate $\overline{R^{\mathrm{SR}, \mathrm{c}}}$ and average probability of detection errors $\bar{\lambda}$ versus the flight period $T$ for different training steps $k_{d}$. In Fig. 6(a), it clearly shows that $\overline{R^{\mathrm{SR}, \mathrm{c}}}$ monotonically increases as $T$ increases, which clearly validates the simulation in Fig. 5. Further, we should notice that, $\overline{R^{\mathrm{SR}, \mathrm{c}}}$ slightly decreases as $k_{d}$ increases. The reason lies in the fact that, a larger $k_{d}$ can train the discriminator better, which can identify the existence of covert information more effectively. In this case, in order to guarantee the same level of probability of detection errors at Eve, the covert rate should be reduced. By contrast, in Fig 6(b), $\bar{\lambda}$ monotonically increases as $T$ increases, which reveals that the covert transmission becomes more difficult to be detected due to the increase of UAV's jamming time. In addition, $\bar{\lambda}$ increases as $k_{d}$ increases. This is because a well trained discriminator can stimulate the generator to generate a promising solution with better covert performance. In general, the above observations indicate that an appropriate $k_{d}$, such as 5 or 10 , can effectively improve the algorithm performance.

\section{Convergence analysis}

In this subsection, we evaluate the convergence performance of the GAN-JTP algorithm by varying training steps and learning rate. Note that, we set the flight period to $T=200 \mathrm{~s}$.

Fig. 7 shows the convergence performance of the proposed algorithm in terms of the loss function values when varying the training steps. Observed at the figure, in the beginning stages, the loss function value of the algorithm converges very quickly. This is benefited from that the gradient descent approach is employed by the GAN-JTP algorithm. In addition, it is shown that the MD-GAN converges within 89 iterations under $k_{d}=1$, 41 iterations under $k_{d}=5$, and 30 iterations under $k_{d}=10$, respectively. The above observation shows that, although the convergence of the generator becomes faster by increasing the training of the discriminator, a larger $k_{d}$ obviously increases the total amount of training and slows down training. Therefore,

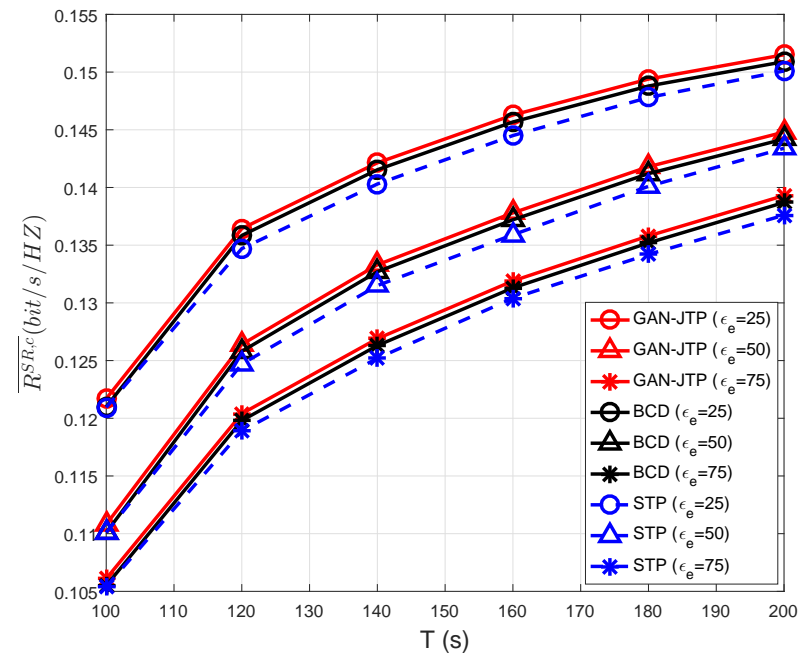

(a) Average covert rate $\overline{R^{\mathrm{SR}, \mathrm{c}}}$ comparison

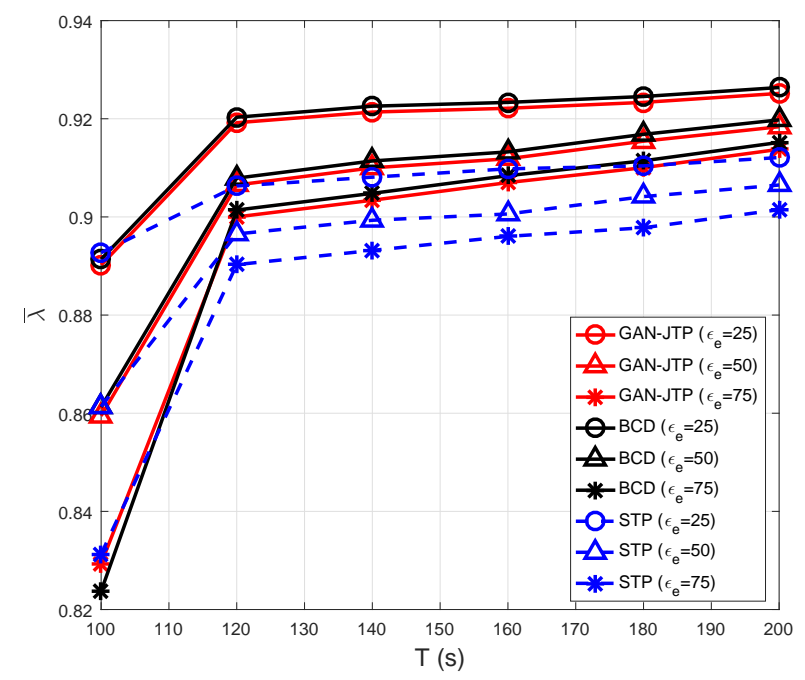

(b) Average probability of detection errors $\bar{\lambda}$ comparison

Fig. 5. Algorithm performance comparison versus the flight period $T$.

in consider of the algorithm performance and expense, we set $k_{d}=5$ in the simulation.

Fig. 8 evaluates the convergence performance of the GANJTP algorithm in terms of the loss function values when employing adaptive learning rate and constant learning rate approaches. Note that, the adaptive learning rate approach varies the learning rate according to the loss function value, shown as (25) and (29), while the constant learning rate approach uses a fixed learning rate of 0.01 . In this figure, both the loss function values fluctuate in the training process. This observation can concretely validate the loss function training rules for the generator and the discriminator in (21) and (22). Specially, the generator and the discriminator are alternatively trained in a competitive manner, and finally approach the Nash equilibrium. Further, it is also observed that, when employing the adaptive learning rate approach described as (25) and (29), the fluctuation range of the loss function value exceeds that 


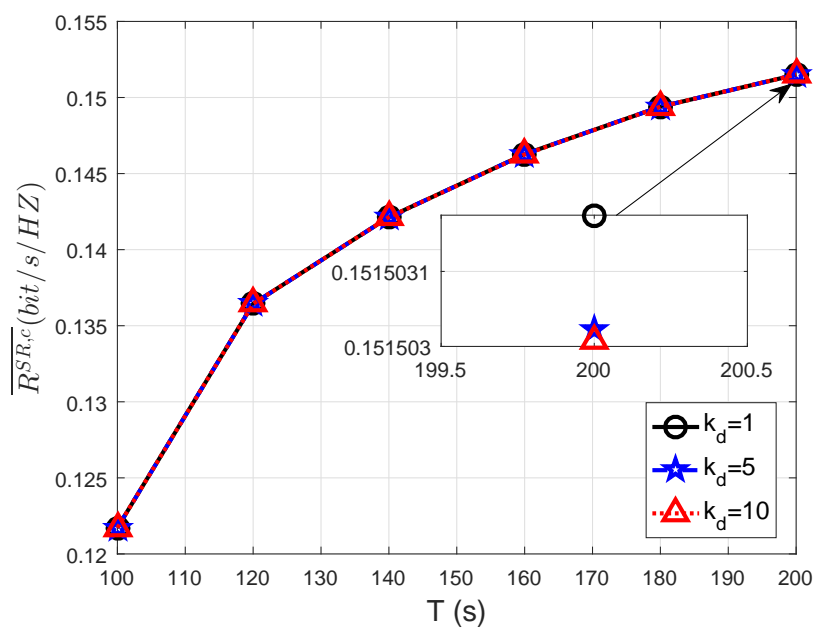

(a) Average covert rate $\overline{R^{\mathrm{SR}, \mathrm{c}}}$ comparison

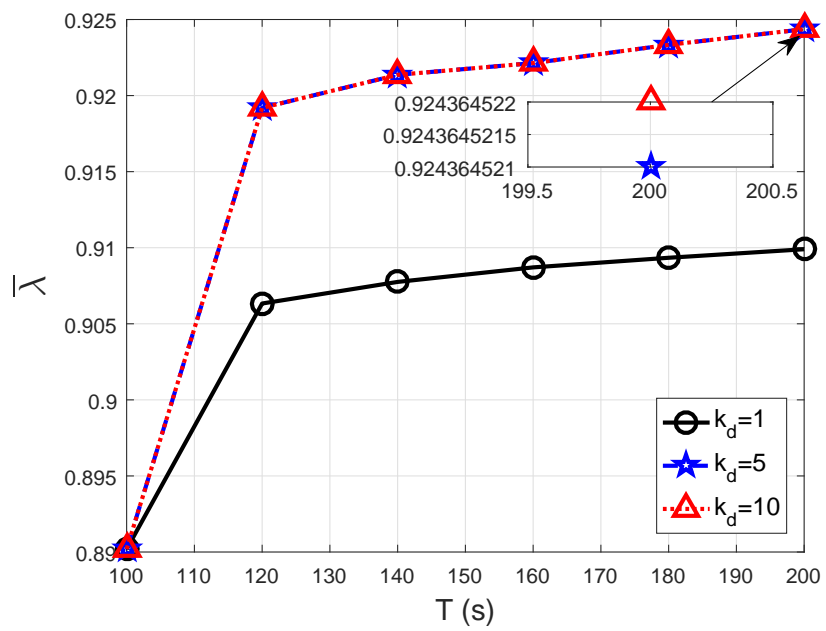

(b) Average probability of detection errors $\bar{\lambda}$ comparison

Fig. 6. Effects of training steps $k_{d}$ on the algorithm performance versus the flight period $T$.

obtained by the constant learning rate approach. In addition, the algorithm with the adaptive learning rate approach converges much faster than that with the constant learning rate approach. In general, the above observations indicate that the GANJTP algorithm has good convergence performance, which will stimulate its practical application.

\section{CONCLUSIONS}

We have investigated the joint UAV's trajectory and power optimization for future cognitive covert communications under the assumptions of knowing the partial CDI and not knowing the detection threshold of the eavesdropper. The analytical scheme has been derived for the multi-objective optimization problem, which has been transformed into an interactive game between the UAV and the eavesdropper. Then the GAN-JTP algorithm has been proposed to train the designed MD-GAN optimization framework with limit amount of labeling data. Our simulation results have shown that, the proposed GANJTP with a rapid convergence speed can attain near-optimal

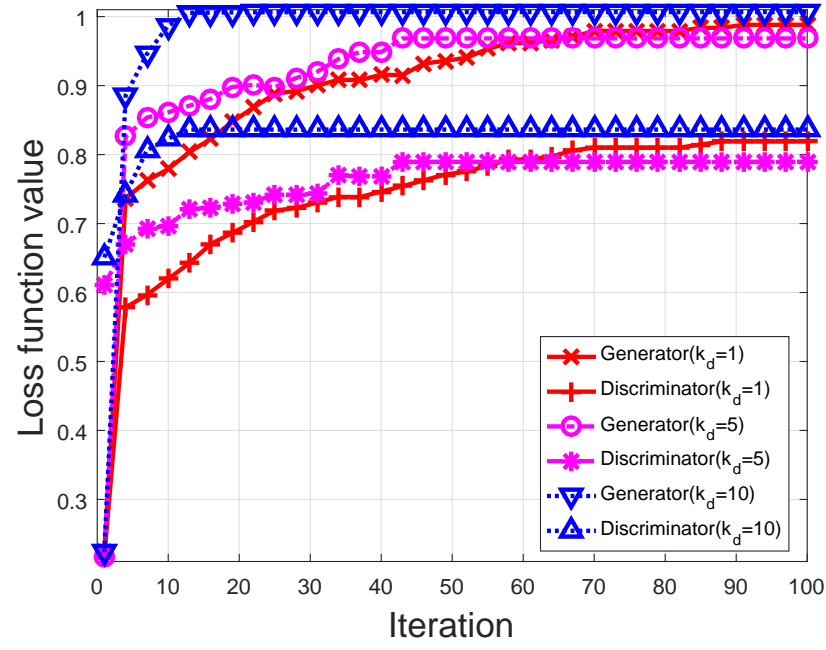

Fig. 7. Effects of training steps $k_{d}$ on the algorithm convergence.

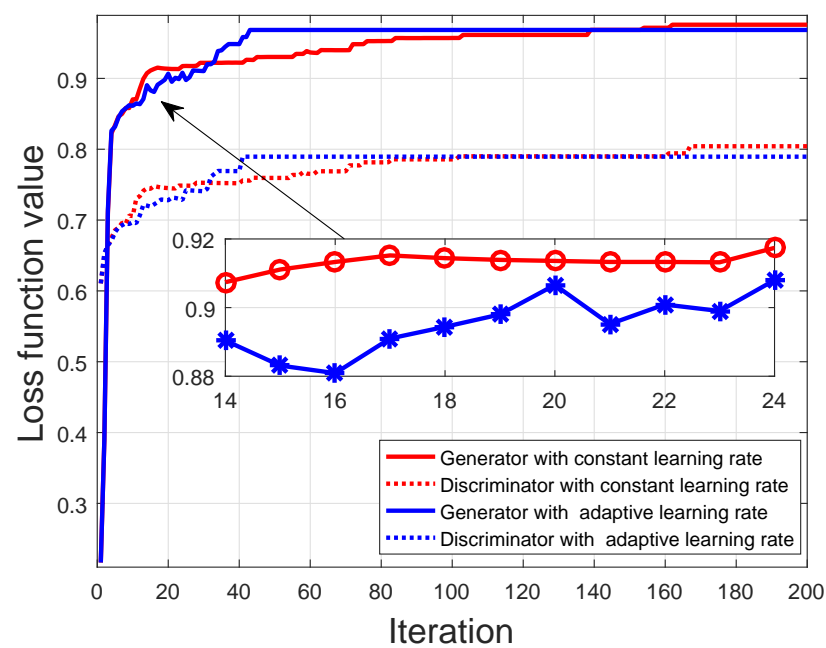

Fig. 8. Effects of learning rate on the algorithm convergence.

solutions of UAV's trajectory and transmit power for the covert communication. Our proposed MD-GAN scheme is a promising candidate for future covert communications in CRNs.

\section{APPENDIX A}

PROOF OF THEOREM 1

Given the probability of detection errors $\lambda[n]$ shown as (14), we find the optimal detection threshold $\tau[n]_{o p}$ for Eve by solving the following optimization problem

$$
\min _{\tau[n]} \lambda[n] .
$$

Then, we prove Theorem 1 in the following cases.

Case 1: When $\tau[n]<\sigma_{\text {Eve }}^{2}, \lambda[n]$ is always a constant $P\left(H_{0}\right)$. It can be easily seen that $\tau[n]_{o p}$ is nonexistent.

Case 2: When $\tau[n] \geq \sigma_{\text {Eve }}^{2}$ and $b[n]=a$, we obtain $\frac{\partial \lambda[n]}{\partial \tau[n]}=\frac{\frac{\tau[n]-\sigma_{\text {Eve }}^{2}}{2 a} P\left(H_{1}\right)-P\left(H_{0}\right)}{2 a} e^{-\frac{\tau[n]-\sigma_{\text {Eve }}^{2}}{2 a}}$, and $\frac{\partial^{2} \lambda[n]}{\partial \tau[n]^{2}}=$

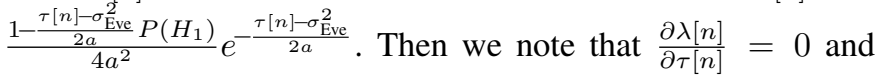


$\frac{\partial^{2} \lambda[n]}{\partial \tau[n]^{2}} \geq 0$ for $\tau[n]=\frac{2 a P\left(H_{0}\right)}{P\left(H_{1}\right)}+\sigma_{\text {Eve }}^{2}$. This indicates that $\frac{2 a P\left(H_{0}\right)}{P\left(H_{1}\right)}+\sigma_{\mathrm{Eve}}^{2}$ is the minimum value. Then, the corresponding minimal probability of detection errors $\lambda[n]_{\min }$ can be obtained as shown in (16).

Case 3: When $\tau[n] \geq \sigma_{\text {Eve }}^{2}$ and $b[n] \neq a$, we get $\frac{\partial \lambda[n]}{\partial \tau[n]}=$ $-\frac{k_{1}}{2 b[n]} e^{-\frac{\tau[n]-\sigma_{\text {Eve }}^{2}}{2 b[n]}}-\frac{k_{2}}{2 a} e^{-\frac{\tau[n]-\sigma_{\text {Eve }}^{2}}{2 a}}$, and $\frac{\partial^{2} \lambda[n]}{\partial \tau[n]^{2}}=\frac{k_{1}}{4 b[n]^{2}} e^{-\frac{\tau[n]-\sigma_{\text {Eve }}^{2}}{2 b[n]}}+$ $\frac{k_{2}}{4 a^{2}} e^{-\frac{\tau[n]-\sigma_{\mathrm{Eve}}^{2}}{2 a}}$. After some simplification, we have $\frac{\partial \lambda[n]}{\partial \tau[n]}=0$ and $\frac{\partial^{2} \lambda[n]}{\partial \tau[n]^{2}} \geq 0$ for $\tau[n]=\frac{2 a b[n]}{a-b[n]} \ln \left[\left(\frac{a}{b[n]}-1\right) \frac{P\left(H_{0}\right)}{P\left(H_{1}\right)}+1\right]+\sigma_{\text {Eve }}^{2}$. Hence, we have the minimum value $\tau[n]^{*}=\frac{2 a b[n]}{a-b[n]} \ln \left[\left(\frac{a}{b[n]}-1\right) \frac{P\left(H_{0}\right)}{P\left(H_{1}\right)}+\right.$ $1]+\sigma_{\text {Eve }}^{2}$. Note that, in the expression of $\tau[n]^{*}$, when we assume that $P\left(H_{1}\right)$ is greater than $P\left(H_{0}\right)$, it is obvious that $\left(\frac{a}{b[n]}-\right.$ 1) $\frac{P\left(H_{0}\right)}{P\left(H_{1}\right)}+1>0$. Then, the corresponding minimal probability of detection errors $\lambda[n]_{\min }$ can be obtained by submitting (15) into (14).

\section{APPENDIX B \\ PROOF OF THEOREM 2}

We first fix the UAV's position to optimize UAV's transmit power, and remove the mobility constraint from optimization problem in (17). Furthermore, the constraint (17b) can be reformulated as follows

$$
\left[\left(\frac{a}{b[n]}-1\right) \frac{P\left(H_{0}\right)}{P\left(H_{1}\right)}+1\right]^{\frac{a}{b[n]-a}} \leq \frac{1-\epsilon-P\left(H_{1}\right)}{P\left(H_{0}\right)-P\left(H_{1}\right)-\frac{a}{b[n]} P\left(H_{0}\right)} .
$$

Then, by introducing the Lambert $\mathrm{W}$ function, (31) can be further equivalently converted as

$$
\frac{a}{b[n]} \leq 1+\frac{W\left\{\frac{P\left(H_{1}\right) \ln \left(\frac{\epsilon+P\left(H_{1}\right)-1}{P\left(H_{1}\right)}\right)}{P\left(H_{0}\right)}\left(\frac{\epsilon+P\left(H_{1}\right)-1}{P\left(H_{1}\right)}\right)^{\frac{P\left(H_{1}\right)}{P\left(H_{0}\right)}}\right\}}{\ln \left(\frac{\epsilon+P\left(H_{1}\right)-1}{P\left(H_{1}\right)}\right)}-\frac{P\left(H_{1}\right)}{P\left(H_{0}\right)},
$$

Finally, according to (6), (11c) and (32), we can get

$$
p^{\mathrm{UAV}}[n]^{*} \leq p^{\mathrm{UAV}}[n] \leq \min \left\{p^{\mathrm{UAV}}[n]^{\max }, P_{\mathrm{UAV}}^{\max }\right\},
$$

where $p^{\mathrm{UAV}}[n]^{\max }=\frac{\frac{p^{\mathrm{PT}}\left|h^{\mathrm{PT}, \mathrm{PR}}\right|^{2}}{{ }_{2} R_{\mathrm{PR}}^{\min }-1}-p^{\mathrm{ST}}\left|h^{\mathrm{ST}, \mathrm{PR}}\right|^{2}-\sigma_{\mathrm{PR}}^{2}}{\left|h^{\mathrm{UAV}, \mathrm{PR}}[n]\right|^{2}}$, and $p^{\mathrm{UAV}}[n]^{*}$ is expressed as (18).

We note that the optimization problem of $\left(\mathcal{P}_{1}\right)$ increases with decreasing $p^{\mathrm{UAV}}[n]$. Therefore, due to the monotonicity, we derive the close-form solution for this UAV's jamming power subproblem as (18).

\section{APPENDIX C \\ PROOF OF THEOREM 3}

We fix the UAV's jamming power $p^{\mathrm{UAV}}[n]$ to optimize the UAV's 3D trajectory. As such, we can transform the constraint in (17b) into (19c), and the constraint in (6) into

$$
d_{\mathrm{UAV}, \mathrm{PR}}[n]^{2} \geq R_{\mathrm{PR}}^{\frac{2}{\alpha_{0}}}
$$

$$
\text { where } R_{\mathrm{PR}}=\frac{\rho p^{\mathrm{UAV}}[n]}{\frac{p^{\mathrm{PT}}\left|h^{\mathrm{PT}, \mathrm{PR}}\right|^{2}}{2^{R_{\mathrm{PR}}^{\min }}-1}-p \mathrm{ST}\left|h^{\mathrm{ST}, \mathrm{PR}}\right|^{2}-\sigma_{\mathrm{PR}}^{2}} \text {. Note that, }
$$

specifies that the UAV's $3 \mathrm{D}$ trajectory is on or outside the sphere centered at the PR with radius $R_{p r}$. As inspired by [23], the feasible region $S_{u}$ in $l$ th $(l \in\{1, \ldots, L\})$ iteration can be approximated by applying a tangent plane as shown in Fig. 9, where $\left(x^{\mathrm{UAV}}[n]^{*(l-1)}, y^{\mathrm{UAV}}[n]^{*(l-1)}, z^{\mathrm{UAV}}[n]^{*(l-1)}\right)$ is the solution for the UAV's location from previous iteration. In this figure, there exists a line $L_{\mathrm{UAV}, \mathrm{PR}}$ passing through the UAV's location from previous iteration and the PR. The tangent plane passes through the intersection point between the line $L_{\mathrm{UAV}, \mathrm{PR}}$ and the sphere centered at the PR with radius $R_{\mathrm{PR}}$, and is perpendicular to the line $L_{\mathrm{UAV}, \mathrm{PR}}$. Note that, the sphere is on one side of the tangent plane, while the feasible region $S_{u}$ is on the other side of the tangent plane.

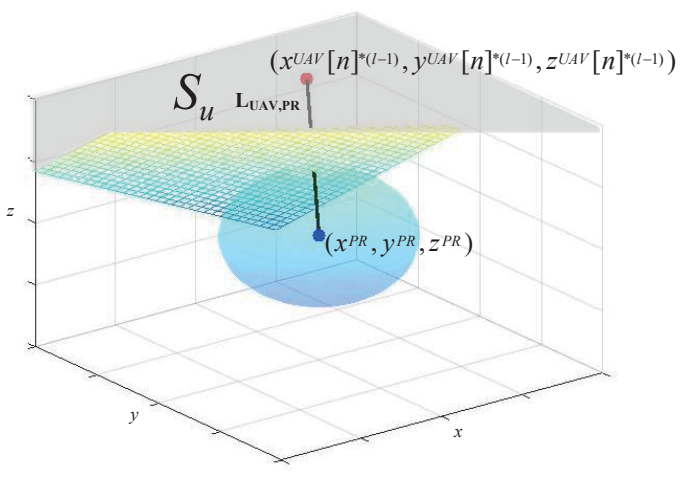

Fig. 9. Schematic for tangential approximation of the feasible region.

Obviously, we note that the optimization problem of $\left(\mathcal{P}_{1}\right)$ increases with increasing $d_{\mathrm{UAV}, \mathrm{SR}}[n]$. Therefore, we remove the power constraint from optimization problem in (17), and the optimization can be further simplified to (19). It is observed that (19) is a constrained convex optimization problem, which can be efficiently solved by the existing convex optimization methods, such as the interior point method [46]. This completes the proof.

\section{REFERENCES}

[1] J. Zhu, Y. Song, D. Jiang, and H. Song, "A new deep-q-learning-based transmission scheduling mechanism for the cognitive internet of things," IEEE Internet of Things Journal, vol. 5, no. 4, pp. 2375-2385, 2018.

[2] H. A. Bany Salameh, S. Almajali, M. Ayyash, and H. Elgala, "Spectrum assignment in cognitive radio networks for internet-of-things delaysensitive applications under jamming attacks," IEEE Internet of Things Journal, vol. 5, no. 3, pp. 1904-1913, 2018.

[3] J. Adu Ansere, G. Han, H. Wang, C. Choi, and C. Wu, "A reliable energy efficient dynamic spectrum sensing for cognitive radio iot networks," IEEE Internet of Things Journal, vol. 6, no. 4, pp. 6748-6759, 2019.

[4] J. Zhang, L. Liu, M. Liu, Y. Yi, Q. Yang, and F. Gong, "Mimo spectrum sensing for cognitive radio-based internet of things," IEEE Internet of Things Journal, vol. 7, no. 9, pp. 8874-8885, 2020.

[5] Y. Zou, "Physical-layer security for spectrum sharing systems," IEEE Transactions on Wireless Communications, vol. 16, no. 2, pp. 1319-1329, 2017.

[6] A. J. Menezes, P. C. van Oorschot, and S. A. Vanstone, Handbook of Applied Cryptography. CRC Press, 2001.

[7] A. Mukherjee, S. A. A. Fakoorian, J. Huang, and A. L. Swindlehurst, "Principles of physical layer security in multiuser wireless networks: A survey," IEEE Communications Surveys Tutorials, vol. 16, no. 3, pp. 1550-1573, 2014.

[8] Y. Zhang, R. Lu, B. Cao, and Q. Zhang, "Cooperative jamming-based physical-layer security of cooperative cognitive radio networks: system model and enabling techniques," IET Communications, vol. 13, no. 5, pp. 539-544, 2019.

[9] B. A. Bash, D. Goeckel, D. Towsley, and S. Guha, "Hiding information in noise: fundamental limits of covert wireless communication," IEEE Communications Magazine, vol. 53, no. 12, pp. 26-31, 2015. 
[10] B. A. Bash, D. Goeckel, and D. Towsley, "Limits of reliable communication with low probability of detection on awgn channels," IEEE Journal on Selected Areas in Communications, vol. 31, no. 9, pp. 1921-1930, 2013.

[11] Z. Liu, J. Liu, Y. Zeng, and J. Ma, "Covert wireless communication in iot network: From awgn channel to thz band," IEEE Internet of Things Journal, vol. 7, no. 4, pp. 3378-3388, 2020 .

[12] P. H. Che, M. Bakshi, and S. Jaggi, "Reliable deniable communication: Hiding messages in noise," in 2013 IEEE International Symposium on Information Theory, 2013, pp. 2945-2949.

[13] L. Wang, G. W. Wornell, and L. Zheng, "Limits of low-probability-ofdetection communication over a discrete memoryless channel," in 2015 IEEE International Symposium on Information Theory (ISIT), 2015, pp. 2525-2529.

[14] C. Gao, B. Yang, X. Jiang, H. Inamura, and M. Fukushi, "Covert communication in relay-assisted iot systems," IEEE Internet of Things Journal, vol. 8, no. 8, pp. 6313-6323, 2021.

[15] S. Yan, B. He, X. Zhou, Y. Cong, and A. L. Swindlehurst, "Delayintolerant covert communications with either fixed or random transmit power," IEEE Transactions on Information Forensics and Security, vol. 14, no. 1, pp. 129-140, 2019.

[16] R. Soltani, D. Goeckel, D. Towsley, B. A. Bash, and S. Guha, "Covert wireless communication with artificial noise generation," IEEE Transactions on Wireless Communications, vol. 17, no. 11, pp. 7252-7267, Nov 2018.

[17] B. He, S. Yan, X. Zhou, and H. Jafarkhani, "Covert wireless communication with a poisson field of interferers," IEEE Transactions on Wireless Communications, vol. 17, no. 9, pp. 6005-6017, Sep. 2018.

[18] K. Shahzad, X. Zhou, S. Yan, J. Hu, F. Shu, and J. Li, "Achieving covert wireless communications using a full-duplex receiver," IEEE Transactions on Wireless Communications, vol. 17, no. 12, pp. 8517-8530, Dec 2018.

[19] Z. Liu, J. Liu, Y. Zeng, and J. Ma, "Covert wireless communications in iot systems: Hiding information in interference," IEEE Wireless Communications, vol. 25, no. 6, pp. 46-52, 2018.

[20] S. Yan, X. Zhou, J. Hu, and S. V. Hanly, "Low probability of detection communication: Opportunities and challenges," IEEE Wireless Communications, vol. 26, no. 5, pp. 19-25, 2019.

[21] Y. Sun, D. Xu, D. W. K. Ng, L. Dai, and R. Schober, "Optimal 3dtrajectory design and resource allocation for solar-powered uav communication systems," IEEE Transactions on Communications, vol. 67, no. 6 , pp. 4281-4298, 2019

[22] C. You and R. Zhang, "3d trajectory optimization in rician fading for uav-enabled data harvesting," IEEE Transactions on Wireless Communications, vol. 18, no. 6, pp. 3192-3207, 2019.

[23] Y. Zhou, P. L. Yeoh, H. Chen, Y. Li, R. Schober, L. Zhuo, and B. Vucetic, "Improving physical layer security via a uav friendly jammer for unknown eavesdropper location," IEEE Transactions on Vehicular Technology, vol. 67, no. 11, pp. $11280-11284,2018$.

[24] A. Li and W. Zhang, "Mobile jammer-aided secure uav communications via trajectory design and power control," China Communications, vol. 15 , no. 8, pp. 141-151, 2018.

[25] A. Li, Q. Wu, and R. Zhang, "Uav-enabled cooperative jamming for improving secrecy of ground wiretap channel," IEEE Wireless Communications Letters, vol. 8, no. 1, pp. 181-184, 2019.

[26] M. Cui, G. Zhang, Q. Wu, and D. W. K. Ng, "Robust trajectory and transmit power design for secure uav communications," IEEE Transactions on Vehicular Technology, vol. 67, no. 9, pp. 9042-9046, 2018.

[27] Y. Cai, Z. Wei, R. Li, D. W. K. Ng, and J. Yuan, "Joint trajectory and resource allocation design for energy-efficient secure uav communication systems," IEEE Transactions on Communications, vol. 68, no. 7, pp. 4536-4553, 2020.

[28] W. Liang, J. Shi, Z. Tie, and F. Yang, "Performance analysis for uavjammer aided covert communication," IEEE Access, vol. 8, pp. 111394 111400,2020

[29] X. Zhou, S. Yan, J. Hu, J. Sun, J. Li, and F. Shu, "Joint optimization of a uav's trajectory and transmit power for covert communications," IEEE Transactions on Signal Processing, vol. 67, no. 16, pp. 4276-4290, 2019.

[30] X. Liao, J. Shi, Z. Li, L. Zhang, and B. Xia, "A model-driven deep reinforcement learning heuristic algorithm for resource allocation in ultradense cellular networks," IEEE Transactions on Vehicular Technology, vol. 69, no. 1, pp. 983-997, 2020.

[31] X. Liao, J. Si, J. Shi, Z. Li, and H. Ding, "Generative adversarial network assisted power allocation for cooperative cognitive covert communication system," IEEE Communications Letters, vol. 24, no. 7, pp. 1463-1467, 2020.
[32] C. Zhong, J. Yao, and J. Xu, "Secure uav communication with cooperative jamming and trajectory control," in IEEE Communications Letters, vol. 23 , no. 2, 2019, pp. 286-289.

[33] J. Li and Y. Han, "Optimal resource allocation for packet delay minimization in multi-layer uav networks," IEEE Communications Letters, vol. 21, no. 3, pp. 580-583, 2017.

[34] S. I. Alnagar, A. M. Salhab, and S. A. Zummo, "Unmanned aerial vehicle relay system: Performance evaluation and 3d location optimization," IEEE Access, vol. 8, pp. $67635-67645,2020$.

[35] X. Liu, Y. Liu, Y. Chen, and L. Hanzo, "Trajectory design and power control for multi-uav assisted wireless networks: A machine learning approach," IEEE Transactions on Vehicular Technology, vol. 68, no. 8, pp. 7957-7969, 2019.

[36] D. Goeckel, S. Vasudevan, D. Towsley, S. Adams, Z. Ding, and K. Leung, "Artificial noise generation from cooperative relays for everlasting secrecy in two-hop wireless networks," IEEE Journal on Selected Areas in Communications, vol. 29, no. 10, pp. 2067-2076, December 2011.

[37] B. A. Bash, D. Goeckel, and D. Towsley, "Covert communication gains from adversarys ignorance of transmission time," IEEE Transactions on Wireless Communications, vol. 15, no. 12, pp. 8394-8405, Dec 2016.

[38] J. Hu, S. Yan, X. Zhou, F. Shu, J. Li, and J. Wang, "Covert communication achieved by a greedy relay in wireless networks," IEEE Transactions on Wireless Communications, vol. 17, no. 7, pp. 4766-4779, July 2018.

[39] J. Hu, S. Yan, F. Shu, and J. Wang, "Covert transmission with a self-sustained relay," IEEE Transactions on Wireless Communications, vol. 18, no. 8, pp. 4089-4102, 2019.

[40] H. Tang, Q. Wu, and B. Li, "An efficient solution for joint power and trajectory optimization in uav-enabled wireless network," IEEE Access, vol. 7, pp. 59 640-59652, 2019.

[41] Q. Wu, Y. Zeng, and R. Zhang, "Joint trajectory and communication design for multi-uav enabled wireless networks," IEEE Transactions on Wireless Communications, vol. 17, no. 3, pp. 2109-2121, 2018.

[42] E. I. Batzelis, I. A. Routsolias, and S. A. Papathanassiou, "An explicit pv string model based on the lambert $w$ function and simplified mpp expressions for operation under partial shading," IEEE Transactions on Sustainable Energy, vol. 5, no. 1, pp. 301-312, 2014.

[43] I. J. Goodfellow, J. Pouget-Abadie, M. Mirza, B. Xu, D. Warde-Farley, S. Ozair, A. C. Courville, and Y. Bengio, "Generative adversarial networks," in Proc. Adv. Neural Inf. Process. Syst., 2014, pp. 2672-2680.

[44] M. T. Hagan, H. B. Demuth, M. H. Beale, and O. D. Jesús, Neural Network Design, 2nd ed. USA: Martin Hagan, 2014.

[45] B. Mao, Z. M. Fadlullah, F. Tang, N. Kato, O. Akashi, T. Inoue, and K. Mizutani, "Routing or computing? the paradigm shift towards intelligent computer network packet transmission based on deep learning," IEEE Transactions on Computers, vol. 66, no. 11, pp. 1946-1960, Nov 2017.

[46] S. Boyd and L. Vandenberghe, Convex Optimization, seven ed. UK: Cambridge University Press, 2009.

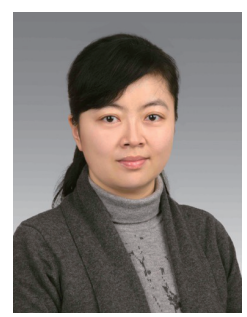

Zan Li received the B.S. degree in communication$\mathrm{s}$ engineering and the M.S. and Ph.D. degrees in communication and information systems from Xidian University, Xi'an, China, in 1998, 2001, and 2006, respectively. She is currently a Professor with the State Key Laboratory of ISN, School of Telecommunication Engineering, Xidian University. Her research interests include wireless communication and signal processing, particularly covert communication, weak signal detection, spectrum sensing, and cooperative communication. She is a fellow of the Institution of Engineering and Technology (IET), the China Institute of Electronics (CIE), and the China Institute of Communications (CIC). She serves as an Associate Editor for IEEE Transactions on Cognitive Communications and Networking and China Communications. 


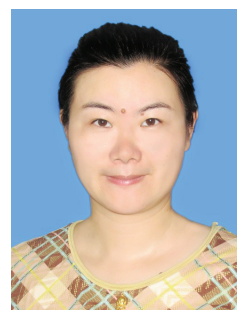

Xiaomin Liao received the B.S. and M.S. degrees from PLA Xi' an Communications Institute in 2006 and 2009, and the Ph.D. degrees in Key Laboratory of Integrated Services Networks (ISN) from Xidian University, Xi'an, China, in 2021. She is currently an Associate Professor in School of Information and Communications, National University of Defense Technology. Her research interests include resource allocation in wireless systems, covert communication, physical layer security, etc.

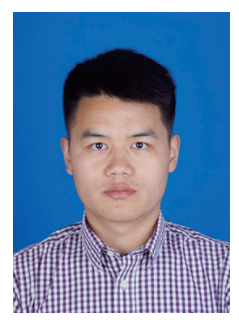

Jia Shi received the M.Sc. and Ph.D. degrees from the University of Southampton, U.K., in 2010 and 2015, respectively. From 2015 to 2017, he was a Research Associate with Lancaster University, U.K. From 2017 to 2018, he became a Research Fellow with 5GIC, University of Surrey, U.K. Since 2018, he has been with Xidian University, China, where he is currently an Associate Professor with the National Key Laboratory of Integrated Services Networks (IS$\mathrm{N})$. His current research interests include resource allocation in wireless systems, covert communications, physical layer security, mmWave communications, and satellite communications. He is also serving as an Associate Editor for Electronic Letters, and an Editor for International Journal of Communications System. He is serving as a Guest Editor for China Communications.

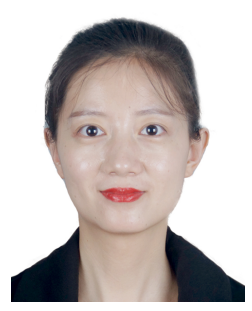

Xuan Xue received the B.E. and Ph.D. degrees from Xidian University, Xi'an, China, in 2010 and 2017, respectively. From 2013 to 2015, she was a Visiting Ph.D. Student with Western University, London, ON, Canada. Since 2018, she has been a Faculty Member with Xidian University, where she is currently a Lecturer with the State Key Laboratory of Integrated Services Networks. Her research interests include massive MIMO, millimeter-wave communications, cooperative communications, and non-orthogonal multiple access.

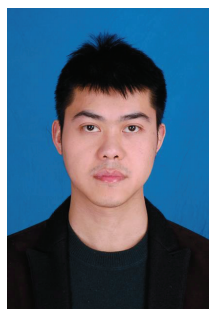

Li Li received the M.Sc. and Ph.D. degree from the Southampton Wireless Group, School of Electronics and Computer Science, University of Southampton, in 2009 and 2013, respectively. He was a Senior Research Assistant with the School of Electronics and Computer Science, University of Southampton, from 2013 to 2014. In 2015, he joined the Provincial Key Laboratory of Information Coding and Transmission, Southwest Jiaotong University, Chengdu, China, serving as an Associate Professor. From 2019, he served as an Editor of IEEE Communications Letters in coding area and was awarded the exemplary editor prize in 2020 His research interests include channel coding, non-orthogonal multiple access technologies, and random access technologies for massive IoT.

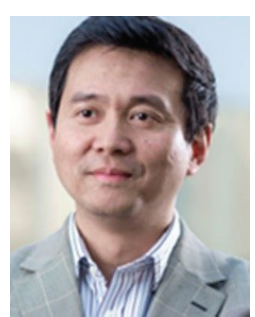

Pei Xiao received the B. Eng, M.Sc. and Ph.D. degree from Huazhong University of Science \& Technology, Tampere University of Technology, Chalmers University of Technology in 1989, 1996, and 2004, respectively. He is a Professor of Wireless Communications at the Institute for Communication Systems, home of 5GIC and 6GIC at the University of Surrey. He is the Technical Manager of 5GIC/6GIC, leading the research team in the new physical layer work area, and coordinating/supervising research activities across all the work areas (www.surrey.ac.uk/5gic/research). Prior to this, he worked at Newcastle University and Queen's University Belfast. He also held positions at Nokia Networks in Finland. He has published extensively in the fields of communication theory, RF and antenna design, signal processing for wireless communications, and is an inventor on over 10 recent 5 GIC patents addressing bottleneck problems in $5 \mathrm{G}$ systems. 